\title{
Adaptive dynamics of saturated polymorphisms
}

\section{Kisdi, Eva}

2016-03

Kisdi , E \& Geritz , S A H 2016 , ' Adaptive dynamics of saturated polymorphisms ' Journal of mathematical biology , vol. 72 , no. 4 , pp. 1039-1079 . DOI: 10.1007/s00285-015-0948-2

http://hdl.handle.net/10138/173477

https://doi.org/10.1007/s00285-015-0948-2

Downloaded from Helda, University of Helsinki institutional repository.

This is an electronic reprint of the original article.

This reprint may differ from the original in pagination and typographic detail.

Please cite the original version. 


\title{
Adaptive dynamics of saturated polymorphisms*
}

\author{
Éva Kisdi \& Stefan A. H. Geritz \\ Department of Mathematics and Statistics \\ University of Helsinki
}

This is the Author Version of an article published in the Journal of Mathematical Biology. The final publication is available at Springer Verlag via https://dx.doi.org/10.1007/s00285-015-0948-2.

Please cite as: Kisdi E. \& S. A. H. Geritz. 2016. Adaptive dynamics of saturated polymorphisms. J. Math. Biol. 72: 1039-1079.

See the end of this file for a correction of Proposition 4.

\section{Abstract}

We study the joint adaptive dynamics of $n$ scalar-valued strategies in ecosystems where $n$ is the maximum number of coexisting strategies permitted by the (generalized) competitive exclusion principle. The adaptive dynamics of such saturated systems exhibits special characteristics, which we first demonstrate in a simple example of a host-pathogenpredator model. The main part of the paper characterizes the adaptive dynamics of saturated polymorphisms in general. In order to investigate convergence stability, we give a new sufficient condition for absolute stability of an arbitrary (not necessarily saturated) polymorphic singularity and show that saturated evolutionarily stable polymorphisms satisfy it. For the case $n=2$, we also introduce a method to construct different pairwise invasibility plots of the monomorphic population without changing the selection gradients of the saturated dimorphism.

Keywords: adaptive dynamics, coevolution, competitive exclusion principle, environmental feedback, saturated community

*This paper is dedicated to Mats Gyllenberg on the occasion of his 60th birthday.

${ }^{\dagger}$ Corresponding author: Éva Kisdi, Department of Mathematics and Statistics, University of Helsinki, PO Box 68, 00014 Helsinki, Finland. Tel.: +358-50-448 0277, e-mail: eva.kisdi@helsinki.fi 
Mathematics Subject Classification (2010) 92D15 92D25 92D40 


\section{Introduction}

Many models of adaptive dynamics start with an initial system harbouring a single resident strategy, and investigate whether natural selection leads to the evolution of higher levels of polymorphism via evolutionary branching (Geritz et al. 1998; for a collection of examples, see http://www.mv.helsinki.fi/home/kisdi/addyn.htm). In this paper, we take the opposite starting point: We consider ecological systems where the maximum number of strategies is already present, and investigate the joint adaptive dynamics of these.

In order to determine the maximum number of coexisting strategies, the concept of environmental feedback variables is of key importance. The environmental feedback variables include all ecological variables such as the population densities of resources, predators, parasites, and mutualists, which together determine the population dynamics of a given strategy. In other words, if the environmental feedback variables were fixed, then the population would grow according to a linear model. All nonlinarities of population growth enter via the dependence of the environmental feedback variables on the population itself (Metz et al. 1992, 1996a; Mylius and Diekmann 1995; Diekmann et al. 1998, 2001, 2003).

The number of strategies coexisting at equilibrium is bounded by the number of the environmental feedback variables (Levin 1970; Geritz et al. 1997; Meszéna et al. 2006). This statement is a generalization of the competitive exclusion principle. In competitive systems, it is well known that the number of species (or strategies) present at equilibrium cannot exceed the number of resources they compete for (excepting the degenerate case of neutral coexistence; MacArthur and Levins 1964). As recognized already by Levin (1970), the same principle applies also outside competitive systems if the resources are replaced with the generalized notion of environmental feedback variables. Note that even if the environmental feedback is infinite dimensional, coexistence of infinitely many species is nongeneric (Gyllenberg and Meszéna 2005).

In this paper, we consider ecological systems with $n$ independent environmental feedback variables containing $n$ strategies. In the main text, we focus on equilibria, where the exclusion principle guarantees that generically no more than $n$ strategies can coexist. We call such $n$-morphic populations saturated polymorphisms.

Non-equilibrium dynamics may increase the dimension of the environmental feedback, but the claim that $n$ dimensions limit coexistence to at most $n$ species remains valid. The exclusion principle and our results therefore extend to non-equilibrium systems with finitedimensional environmental feedback as well. More details on this are given in Appendix A.

The adaptive dynamics of saturated polymorphisms bear some particular characteristics. Obviously, evolutionary branching is impossible in a saturated polymorphism. Models where $n=1$ and the population growth rate is a uniformly monotonic function of 
the single environmental feedback variable are optimization models, the special properties of which are well understood (Metz et al. 1996b, 2008; Meszéna et al. 2001; Gyllenberg and Service 2011; Gyllenberg et al. 2011; Metz and Geritz, in press). One may readily expect that these special properties generalize in some form also to saturated polymorphisms with $n>1$, but so far only sporadic observations exist for the latter. In a model with two environmental feedback variables, Svennungsen and Kisdi (2009) pointed out that a dimorphic singularity of scalar strategies is convergence stable if it is evolutionarily stable; the same holds in optimization models. It may be noticed in several published models that the isoclines of zero selection gradients in saturated dimorphisms intersect at right angles at the dimorphic singularities (see e.g. Figure 1 of Geritz and Kisdi 2000; Figure 1 of Mathias and Kisdi 2002; Figure 3 of Svennungsen an Holen 2007; Figure 3 of Svennungsen and Kisdi 2009; Figure 4 of Boldin and Kisdi 2012; Figure 2 of Weigang and Kisdi 2015), which hints at further unexplored properties of saturated polymorphisms. It is the aim of this paper to study the specific properties of the adaptive dynamics of saturated polymorphisms.

In section 2 of this paper, we use a simple example to demonstrate a number of features exhibited by the evolution of saturated polymorphisms. Our main results, in section 3, are given in a series of propositions that characterize the adaptive dynamics of saturated polymorphisms in general. When considering convergence stability, we extend the conditions for the strongest form of stability, called absolute stability, to arbitrary $k$-morphic singularities (see Appendix B) before applying them to saturated polymorphisms. In Section 4, we explore some connections between the adaptive dynamics of saturated polymorphisms and of populations one level of polymorphism lower. For the case of $n=2$, we also introduce a construction method to obtain different pairwise invasibility plots without changing the selection gradients of the saturated dimorphism; this illustrates how the adaptive dynamics of saturated polymorpisms are constrained relative to lower levels of polymorpism.

\section{A motivating example}

Before turning to our main results, we present an example in order to illustrate the phenomena the generality of which we are going to prove. In this section, we consider a model with $n=2$ environmental feedback variables. This implies that at most two strategies can coexist at equilibrium, i.e., a dimorphic population is saturated; and since the coevolution of two strategies is easy to visualize, we can demonstrate the properties of adaptive dynamics in saturated communities graphically.

We take the evolution of pathogen virulence under selective predation as an example. The model we investigate in this section is closely related to the model studied by Morozov and Best (2012), who have demonstrated evolutionary branching of pathogens under selective predation, and to the model of Kisdi et al. (2013), who analyzed eco-evolutionary 
cycles in this system. The model is the same as what Kisdi (2015) used as an example to illustrate how one can construct trade-off functions to obtain evolutionary branching or any other outcome of adaptive dynamics in monomorphic resident populations. The evolution of a dimorphic population in this model has, however, not yet been explored.

\subsection{The specific model}

We consider a host population that harbours $m$ strains of the pathogen (we shall focus on $m=2$ ). The population density $I_{j}$ of hosts infected with the $j$ th strain obeys the dynamics

$$
\frac{d I_{j}}{d t}=\left[\beta\left(\alpha_{j}\right) S-\left(\alpha_{j}+\mu+\nu\right)-\left(c+\phi\left(\alpha_{j}\right)\right) P\right] I_{j} \quad \text { for } j=1, \ldots, m .
$$

Here $\alpha_{j}$ is the virulence of strain $j$, which also determines its transmission rate $\beta\left(\alpha_{j}\right) . \mu$ and $\nu$ are the natural mortality rate and the rate of recovery, respectively. An infected host is subject to predation at rate $c+\phi\left(\alpha_{j}\right)$, where $c$ is the baseline predation rate that also applies to healthy hosts and $\phi\left(\alpha_{j}\right)$ is the excess predation rate that depends on the virulence of the strain. The model has two environmental feedback variables determined by the resident system, the population density of susceptible hosts $(S)$ and the density of predators $(P)$.

The dynamics of $S$ and $P$ are described by additional equations, which we shall refer to as the embedding model:

$$
\begin{aligned}
\frac{d S}{d t} & =B(N)-(\mu+c P) S-\sum_{j=1}^{m} \beta\left(\alpha_{j}\right) I_{j} S+\nu \sum_{j=1}^{m} I_{j} \\
\frac{d P}{d t} & =\left[\gamma c N+\gamma \sum_{j=1}^{m} \phi\left(\alpha_{j}\right) I_{j}+\theta \zeta Z-\delta\right] P \\
\frac{d Z}{d t} & =[\rho(Z)-\zeta P] Z
\end{aligned}
$$

where $N=S+\sum_{j=1}^{m} I_{j}$ is total host density. In (2a), hosts are born susceptible at the population birth rate $B(N)$, and susceptible hosts die at the per capita rate given by the sum of the background mortality rate, $\mu$, and mortality due to predation, $c P$. Infection and recovery occur as described above, and recovered individuals return to the susceptible class (no acquired immunity). In (2b), we assume that predators convert all hosts they capture into predator offspring at a conversion factor $\gamma$. The predator has also an alternative prey, with density $Z$, which is captured at a rate $\zeta$ and converted with a factor $\theta$. The predators die at a constant rate $\delta$. Finally, (2c) describes the dynamics of the alternative prey, which has a density-dependent per capita growth rate $\rho(Z)$ in absence 
of predation.

In this example, we assume that the full ecological dynamics in equations (1)-(2) attain a unique stable equilibrium. In the numerical examples presented in Figures 1 and 2 , we have confirmed this by checking all equilibria and their Jacobians in monomorphic $(m=1)$ and dimorphic $(m=2)$ resident populations over the part of the trait space shown. We remark, however, that the results of section 2.2 would not change even if the system had non-equilibrium dynamics (see Appendix A). The numerical analysis was done in Mathematica 9.0.1 (Wolfram Research). Since the equilibrium equations derived from (1) can generically not be satisfied for more than two strains of the pathogen at positive densities, the system is saturated with $m=2$ strains.

If a new mutant strain $\alpha_{m u t}$ appears at an infinitesimally low density in the resident system at equilibrium, then, from equation (1), initially the mutant grows exponentially at the rate given by its invasion fitness,

$$
r\left(\alpha_{m u t}, \hat{S}, \hat{P}\right)=\beta\left(\alpha_{m u t}\right) \hat{S}-\left(\alpha_{m u t}+\mu+\nu\right)-\left(c+\phi\left(\alpha_{m u t}\right)\right) \hat{P}
$$

where $\hat{S}$ and $\hat{P}$ are the equilibrium densities of susceptibles and predators, respectively. Assume that mutations have small phenotypic effect (i.e., a mutant of strain $j$ is characterized with a virulence $\alpha_{m u t}=\alpha_{j}+d \alpha$ with a small mutational effect $d \alpha$ ). Then by a series mutations that invade and substitute the former resident, the $j$ th strain evolves towards higher or lower virulence according to whether its selection gradient,

$$
D_{j}=\left.\frac{\partial r}{\partial \alpha_{m u t}}\right|_{\alpha_{m u t}=\alpha_{j}}=\beta^{\prime}\left(\alpha_{j}\right) \hat{S}-1-\phi^{\prime}\left(\alpha_{j}\right) \hat{P}
$$

is positive or negative (Geritz et al. 1998; Geritz 2005).

\subsection{Dimorphic evolution}

Let us now focus on the saturated case $m=2$. Setting $d I_{i} / d t=0$ for $i=1,2$ in (1) and solving for $S$ and $P$, we obtain the densities at the interior equilibrium for any given pair of strains, $\alpha_{1}$ and $\alpha_{2}$, as

$$
\begin{aligned}
& \hat{S}\left(\alpha_{1}, \alpha_{2}\right)=\frac{g\left(\alpha_{2}\right) h\left(\alpha_{1}\right)-g\left(\alpha_{1}\right) h\left(\alpha_{2}\right)}{g\left(\alpha_{2}\right) \beta\left(\alpha_{1}\right)-g\left(\alpha_{1}\right) \beta\left(\alpha_{2}\right)} \\
& \hat{P}\left(\alpha_{1}, \alpha_{2}\right)=\frac{h\left(\alpha_{2}\right) \beta\left(\alpha_{1}\right)-h\left(\alpha_{1}\right) \beta\left(\alpha_{2}\right)}{g\left(\alpha_{1}\right) \beta\left(\alpha_{2}\right)-g\left(\alpha_{2}\right) \beta\left(\alpha_{1}\right)}
\end{aligned}
$$

where $g(\alpha)=c+\phi(\alpha)$ and $h(\alpha)=\alpha+\mu+\nu$. Substituting these into (4), we immediately obtain the selection gradients $D_{1}\left(\alpha_{1}, \alpha_{2}\right)$ and $D_{2}\left(\alpha_{1}, \alpha_{2}\right)$ directly as functions of the trait values $\alpha_{1}$ and $\alpha_{2}$. Note that for some trait values, $\hat{S}\left(\alpha_{1}, \alpha_{2}\right), \hat{P}\left(\alpha_{1}, \alpha_{2}\right)$ or the equilibrium densities $\hat{I}_{1}\left(\alpha_{1}, \alpha_{2}\right), \hat{I}_{2}\left(\alpha_{1}, \alpha_{2}\right), \hat{Z}\left(\alpha_{1}, \alpha_{2}\right)$ obtained from the embedding model may be 
negative, i.e., not all points $\left(\alpha_{1}, \alpha_{2}\right)$ correspond to biologically feasible dimorphisms. Nevertheless, we extend the definition of the selection gradient to all points where $\hat{S}\left(\alpha_{1}, \alpha_{2}\right)$ and $\hat{P}\left(\alpha_{1}, \alpha_{2}\right)$ in (5) exist, and investigate which subset represents biologically feasible systems in section 2.3 .

In Figure 1, the horizontal and vertical arrows show the direction of evolution of $\alpha_{1}$ and $\alpha_{2}$ (should the dimorphism be feasible) according to the selection gradients $D_{1}\left(\alpha_{1}, \alpha_{2}\right)$ and $D_{2}\left(\alpha_{1}, \alpha_{2}\right)$, respectively. From the selection gradients, we obtain the isoclines of strain $j$, i.e., the curves defined by $D_{j}\left(\alpha_{1}, \alpha_{2}\right)=0$ (thick lines in Figure 1). The dimorphic singularity, where directional evolution comes to a halt in both strains, corresponds to the intersection of the isoclines away from the diagonal $\alpha_{2}=\alpha_{1}$. Furthermore, a community on the $j$ th isocline is uninvadable with respect to mutants of strain $j$ if

$$
\left.\frac{\partial^{2} r\left(\alpha_{m u t}, \hat{S}\left(\alpha_{1}, \alpha_{2}\right), \hat{P}\left(\alpha_{1}, \alpha_{2}\right)\right)}{\partial \alpha_{m u t}^{2}}\right|_{\alpha_{m u t}=\alpha_{j}}<0
$$

(Geritz et al. 1998; continuous versus dashed isoclines in Figure 1). The intersection of uninvadable isoclines corresponds to an evolutionarily stable dimorphic singularity. The shading in Figure 1 shows the equilibrium values $\hat{S}\left(\alpha_{1}, \alpha_{2}\right)$ in the left panels and $\hat{P}\left(\alpha_{1}, \alpha_{2}\right)$ in the right panels.

Figure 1 has several conspicuous features:

- At the dimorphic singularity (i.e., away from the diagonal $\alpha_{2}=\alpha_{1}$ ), the isoclines intersect at right angles such that near their intersection, the $\alpha_{1}$-isocline is perpendicular to the $\alpha_{1}$-axis and the $\alpha_{2}$-isocline is perpendicular to the $\alpha_{2}$-axis.

- A strain evolves towards its isocline if and only if the isocline is uninvadable (uninvadable isoclines are drawn with solid lines in Figure 1). An evolutionarily stable dimorphic singularity is also convergence stable and vice versa (note that this is generally not the case under frequency-dependent selection (Eshel 1983), but it is so in Figure 1).

- The isoclines trace out special lines on the surfaces of environmental feedbacks. For each feedback variable, it holds that the $\alpha_{1}$-isocline follows the points where the feedback has a critical point with respect to changing $\alpha_{1}$ (i.e., looking horizontally), and the same applies to the $\alpha_{2}$-isocline with respect to changing $\alpha_{2}$ (looking vertically).

- The equilibrium value of each feedback variable at the $\alpha_{1}$-isocline equals the limit of the same feedback variable to the diagonal at the same $\alpha_{1}$-coordinate (directly below/above the isocline, shown at two points by the vertical guide lines in the bottom panels of Figure 1); and the equilibrium value of each feedback variable at the $\alpha_{2}$-isocline equals to the limit of the same feedback variable to the diagonal 

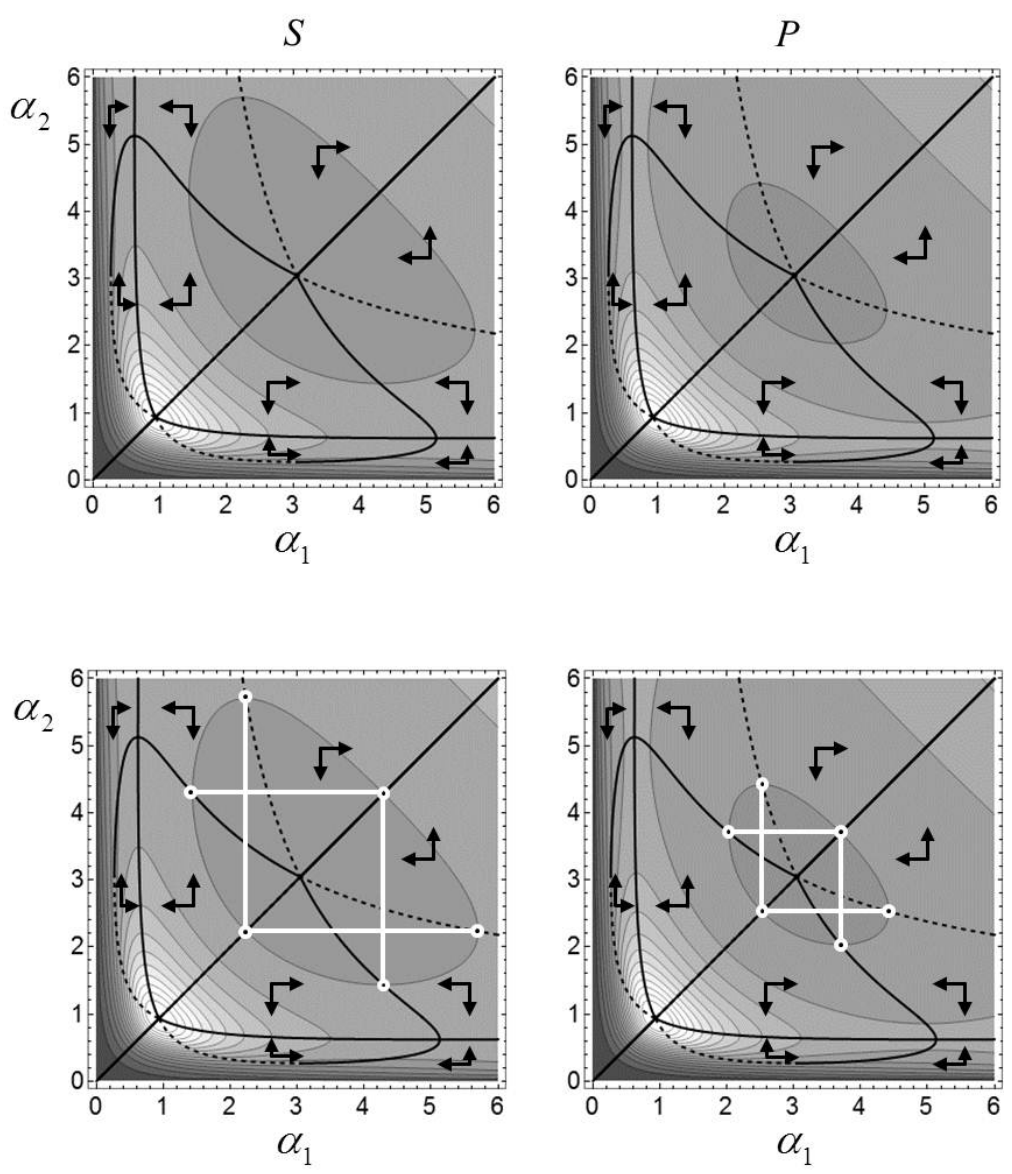

Figure 1: Trait evolution plot obtained from equation (1). Horizontal and vertical arrows show the direction of evolution in $\alpha_{1}$ and $\alpha_{2}$, respectively, according to the sign of (4). The directions change across the isoclines and across the diagonal $\alpha_{2}=\alpha_{1}$ (thick lines). Segments of the isoclines where condition (6) holds and where it does not hold are drawn with continuous and dashed lines, respectively. The grey shading shows the equilibrium values of the feedback variables, $\hat{S}\left(\alpha_{1}, \alpha_{2}\right)$ in the left panels and $\hat{P}\left(\alpha_{1}, \alpha_{2}\right)$ in the right panels (light shading represents high values). The bottom panels contain white guide lines to highlight that the equilibrium values of the environmental feedback variables at points on the $\alpha_{1^{-}}\left(\alpha_{2^{-}}\right)$isoclines equal their limiting values at the diagonal at the same $\alpha_{1^{-}}\left(\alpha_{2^{-}}\right)$coordinate; otherwise the bottom panels are identical to the top panels. The plots are symmetric on the diagonal because the labelling of the two strains is arbitrary. Functions and parameters: $\beta(\alpha)=\alpha^{0.15}, \phi(\alpha)=1-e^{-\alpha}, \mu=1$, $\nu=0.1, c=2$.

at the same $\alpha_{2}$-coordinate (directly to the right/left of the isocline, shown at two points by the horizontal guide lines).

- Each environmental feedback has a saddle point at the dimorphic ESS.

- Each environmental feedback attains critical points where the isoclines cross the diagonal $\alpha_{2}=\alpha_{1}$. 
These features constrain the evolution of the saturated dimorphic system, and also indicate a strong link between the adaptive dynamics of the saturated dimorphism and the geometry of the ecological equilibria of the feedback variables as functions of the resident trait values. In section 3, we prove these statements in suitably generalized forms for arbitrary saturated polymorphisms.

It is important to notice that all information contained in Figure 1 has been obtained from equation (1) and is therefore completely independent of the embedding model in (2). Changing the details of the embedding model will thus not affect the position and evolutionary stability of isoclines and dimorphic singularities. This implies very considerable freedom in the ecological model. One could not only change the values of parameters and the shapes of functions that only occur in the embeddig model (such as the birth rate function $B$ or the dynamics of the alternative prey given by the function $\rho$ ), but also add (or remove) alternative prey species; introduce competitors or other predators that affect the alternative prey species, thereby building more complex food webs; introduce other pathogens infecting either the focal host (with full cross-immunity) or other species in the food web; etc. All these changes would not affect Figure 1.

The only piece of relevant information that is not given in Figure 1, and which depends on the embedding model, is the positivity and stability of the ecological equilibrium. In particular, at some points in Figure 1, the nontrivial equilibrium $\hat{I}_{1}$ or $\hat{I}_{2}$ will be negative, i.e., some points will be outside the area of coexistence of the two strains. Assuming that the ecological attractor is unique for each pair of strains, the area of coexistence can be derived from the adaptive dynamics of monomorphic populations.

\subsection{Monomorphic evolution and the area of coexistence}

If only a single resident strain is present $(m=1)$, then equation (1) is not sufficient to determine both $\hat{S}$ and $\hat{P}$. In monomorphic populations, the equilibrium values of the environmental feedbacks, and therefore also the invasion fitness in (3) depend on the embedding model. Let $s_{\alpha}\left(\alpha_{m u t}\right)$ denote the invasion fitness of a rare mutant $\alpha_{m u t}$ in the monomorphic resident population of strain $\alpha$, i.e., $s_{\alpha}\left(\alpha_{m u t}\right)=r\left(\alpha_{m u t}, \hat{S}, \hat{P}\right)$ with $\hat{S}$ and $\hat{P}$ taking the equilibrium values of the resident system of strain $\alpha$. The strains $\alpha_{1}$ and $\alpha_{2}$ are mutually invasible if $s_{\alpha_{1}}\left(\alpha_{2}\right)>0$ and $s_{\alpha_{2}}\left(\alpha_{1}\right)>0$. Assuming that the ecological attractor is unique, the two strains coexist at positive densities if and only if they are mutually invasible. The area of coexistence can therefore be constructed by taking the sign plot of of the invasion fitness (known as pairwise invasibility plot), and combining it with its mirror image on the diagonal $\alpha_{2}=\alpha_{1}$; the overlapping positive parts give the area of mutual invasibility (Geritz et al. 1998).

Figure 2 illustrates how the pairwise invasibility plot (left panels) and the area of coexistence (white area in the right panels) change with changing some parameters of the 
embedding model. Since the dimorphic invasion fitness is not affected by the embedding model, the dimorphic selection gradients, the isoclines, and the dimorphic singularity remain the same; these give the identical "backdrop" in each of the right panels of Figure 2. We have verified in these examples that all monomorphic populations in the range of $\alpha$ shown and all dimorphic populations in the area of coexistence have a unique positive equilibrium of the system (1)-(2), and that this equilibrium is asymptotically stable; and that pairs of strains outside the area of coexistence have no positive ecological equilibria.

The embedding model influences whether a dimorphic singularity is in the area of coexistence (i.e., whether it represents a biologically feasible system) and whether it can be reached from an initially monomorphic population via evolutionary branching. Figure 2 demonstrates this. The three examples in panels (a), (b), and (c) differ only in $\zeta$, the predator's capture rate towards its alternative prey; this seemingly minor detail can however make a significant difference for evolution. In panel (a), the pairwise invasibility plot exhibits three monomorphic singularities, a convergence stable ESS, a repellor, and an evolutionary branching point. After evolutionary branching, the two strains can evolve to the dimorphic evolutionarily stable singularity at the intersection of the isoclines. (Note that if the less virulent strain evolves too fast, then the trajectory can also leave the area of coexistence so that the more virulent strain goes extinct; the remaining strain then evolves to the ESS.) In panel (b), there is no evolutionary branching point, but the dimorphic evolutionarily stable singularity is still in the area of coexistence, so that it can be reached by an initially dimorphic population. In panel (c), the dimorphic singularity is not in the area of coexistence. In an initially dimorphic population, the more virulent strain evolves upwards until the trajectory leaves the area of coexistence and the less virulent strain goes extinct. The remaining strain then evolves to the highest monomorphic singularity, which is an ESS.

Panels (a), (d), and (e) of Figure 2 differ only in the parameters of the birth rate function $B$. In panels (a) and (d), there is an evolutionary branching point, but in panel (d), the dimorphic singularity is not in the area of coexistence; after branching in (d), the dimorphic population evolves until the more virulent strain goes extinct. In panel (e), the area of coexistence cannot be reached from monomorphic populations, and evolution in an initially dimorphic population leads to the extinction of the more virulent strain.

In section 4.2 , we discuss how the shape of the pairwise invasibility plot can be influenced by choosing a function of the embedding model appropriately. 

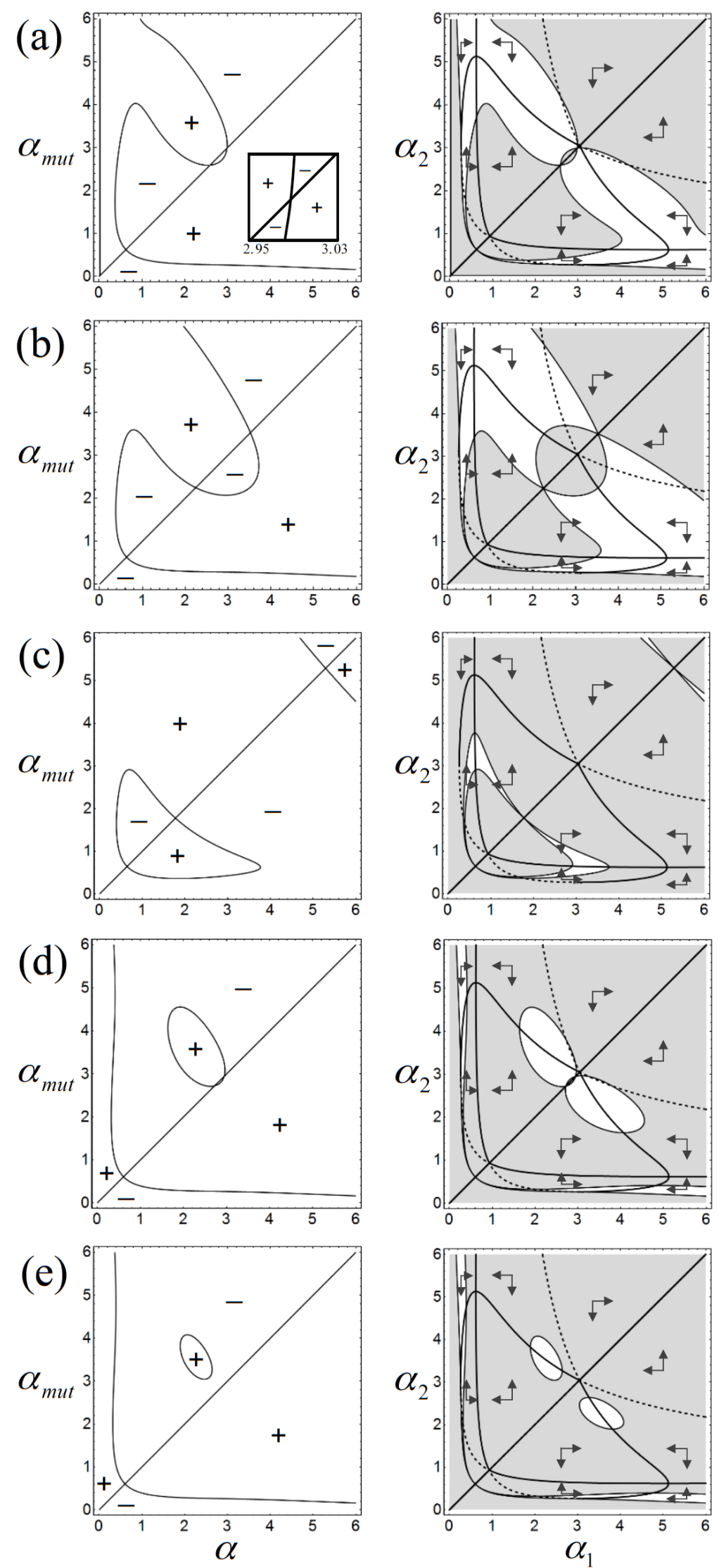
Figure 2 (previous page): Pairwise invasibility plots (left panels) and the area of coexistence overlaid the trait evolution plot (right panels). In the pairwise invasibility plots, "+" and "-" mark the sign of the invasion fitness; in (a), the inset shows that the rightmost singularity is an evolutionary branching point. In the trait evolution plots, the white parts are the area of coexistence; other notations as in Figure 1. Notice that the selection gradients and isoclines are identical across the right panels (they form a constant "backdrop" in the figures) and identical to Figure 1. The only difference is the area of coexistence, which derives from the corresponding pairwise invasibility plot. Functions and parameters in equation (1) as in Figure 1; those in equation $(2), B(N)=\left(b_{0}-a N\right) N ; \rho(Z)=\rho_{0}(1-Z / K) ; \gamma=0.00136 ; \theta=0.005 ; \delta=1 ; \rho_{0}=40$; $K=200$ and (a) $b_{0}=27.45, a=0.001779, \zeta=0$; (b) $b_{0}=27.45, a=0.001779, \zeta=0.5 ;(\mathbf{c})$ $b_{0}=27.45, a=0.001779, \zeta=1.25 ;$ (d) $b_{0}=41.25, a=0.0556, \zeta=0 ;(\mathbf{e}) b_{0}=41, a=0.0556$, $\zeta=0$.

\section{General model}

Consider a model where $n$ environmental feedback variables, $E_{1}, \ldots, E_{n}$, determine the invasion fitness $r\left(x, E_{1}, \ldots, E_{n}\right)$ of a strategy characterized with a scalar trait value $x \in$ $\mathbb{X} \subseteq \mathbb{R}$. If $n$ resident strategies $x_{1}, \ldots, x_{n}$ coexist at an equilibrium, then each must have zero growth rate such that

$$
\begin{aligned}
r\left(x_{1}, E_{1}, \ldots, E_{n}\right) & =0 \\
& \vdots \\
r\left(x_{n}, E_{1}, \ldots, E_{n}\right) & =0
\end{aligned}
$$

The $n$ resident strategies are embedded in an ecosystem consisting of the environmental feedbacks such as resources and predators, and possibly other species that interact with the feedbacks and with each other. The equilibrium conditions of the entire ecosystem are given by the $n$ equations in (7) that determine the equilibrium values of the $n$ feedback variables, together with $n+k$ equations of the form

$$
F_{i}\left(x_{1}, \ldots, x_{n}, E_{1}, \ldots, E_{n}, N_{1}, \ldots, N_{n}, Z_{1}, \ldots, Z_{k}\right)=0 \quad \text { for } i=1, \ldots, n+k
$$

for the equilibrium values of $N_{1}, \ldots, N_{n}$, the population densities of the $n$ resident strategies, and for the equilibrium values of $Z_{1}, \ldots, Z_{k}$, which represent all other population dynamical variables of the model. The latter may be population densities of other species, such as the alternative prey in the example of section 2 , where $F_{i}(i=1,2,3)$ are the right hand sides of the three equations in (2). The resident strategies may also have structured populations, in which case $N_{i}$ is the total population size of strategy $x_{i}$ (or any suitable norm of its population vector), and the variables describing its population structure (e.g. the relative frequencies of $x_{i}$-individuals in various demographic states) are listed among the variables $Z_{1}, \ldots, Z_{k}$. The same applies when the environmental feedbacks are biological species with structured populations; for example, if the juveniles of a species are consumed by the residents but the adults are not, then the juvenile density is one 
of the environmental feedback variables, whereas the adult density appears as one of the variables $Z_{1}, \ldots, Z_{k}$. Note that $N_{1}, \ldots, N_{n}$ may also appear in the feedback variables. For example, the weighted sum $\sum_{i=1}^{n} w\left(x_{i}\right) N_{i}$ may be one of the feedback variables, say $E_{j}$, in which case (8) contains the equation $E_{j}-\sum_{i=1}^{n} w\left(x_{i}\right) N_{i}=0$.

The central question of this paper is how much information we can extract from only the invasion fitness function $\left(x, E_{1}, \ldots, E_{n}\right) \mapsto r\left(x, E_{1}, \ldots, E_{n}\right)$ (and therefore from equations (7)) about the coevolution of the trait values $x_{1}, \ldots, x_{n}$ of the $n$ coexisting strategies. In other words, we investigate to what extent the adaptive dynamics of a saturated polymorphism is independent of all the details (and this typically includes many details) that appear only as part of the embedding model in equations (8).

We make the following assumptions about the invasion fitness and the embedding model:

(A1) The invasion fitness $r$ is twice continuously differentiable with respect to all its arguments, and the functions $F_{1}, \ldots, F_{n+k}$ are continuously differentiable with respect to all their arguments.

(A2) Equations (7)-(8) have an isolated solution for the equilibrium values of the feedback variables $E_{1}, \ldots, E_{n}$ and of $N_{1}, \ldots, N_{n}, Z_{1}, \ldots, Z_{k}$ when $\left(x_{1}, \ldots, x_{n}\right)$ is in an open set $U_{1} \subseteq \mathbb{X}^{n}$

Note that $x_{1}, \ldots, x_{n}$ must all be different for $\left(x_{1}, \ldots, x_{n}\right) \in U_{1}$. For simplicity, we shall treat the equilibrium as if it were unique, but the propositions can be extended to multiple equilibria using the results of Geritz et al. (2002). For non-equilibrium population dynamics, see Appendix A.

(A3) If $N_{j}=0$, then the functions $F_{1}, \ldots, F_{n+k}$ are constant with respect to $x_{j}$.

The last assumption corresponds to the biological fact that if a strategy is not present, then its trait value does not influence the dynamics of the system.

We adopt the notation $\partial_{j} r\left(x, E_{1}, \ldots, E_{n}\right)$ for the partial derivative of $r$ with respect to its $j$ th argument evaluated at the point $\left(x, E_{1}, \ldots, E_{n}\right)$, and we use double subscripts to denote second partial derivatives.

Let $\hat{E}_{1}\left(x_{1}, \ldots, x_{n}\right), \ldots, \hat{E}_{n}\left(x_{1}, \ldots, x_{n}\right)$ denote the solution of equations (7) for the equilibrium values of the feedback variables for $\left(x_{1}, \ldots, x_{n}\right) \in U_{1}$. Since the trait values $x_{1}, \ldots, x_{n}$ fully determine the feedbacks $\hat{E}_{1}, \ldots, \hat{E}_{n}$, they also determine the selection gradients of the $n$ strategies,

$$
\begin{gathered}
\partial_{1} r\left(x_{1}, \hat{E}_{1}\left(x_{1}, \ldots, x_{n}\right), \ldots, \hat{E}_{n}\left(x_{1}, \ldots, x_{n}\right)\right) \\
\vdots \\
\partial_{1} r\left(x_{n}, \hat{E}_{1}\left(x_{1}, \ldots, x_{n}\right), \ldots, \hat{E}_{n}\left(x_{1}, \ldots, x_{n}\right)\right)
\end{gathered}
$$


Hence at each point $\left(x_{1}, \ldots, x_{n}\right) \in U_{1}$, we can say whether the $j$ th strategy evolves towards higher or lower trait values $\left(\partial_{1} r\left(x_{j}, \hat{E}_{1}, \ldots, \hat{E}_{n}\right)\right.$ is positive or negative). In particular, the $n-1$ dimensional manifold in $\mathbb{X}^{n}$ determined by

$$
\partial_{1} r\left(x_{j}, \hat{E}_{1}\left(x_{1}, \ldots, x_{n}\right), \ldots, \hat{E}_{n}\left(x_{1}, \ldots, x_{n}\right)\right)=0
$$

corresponds the $x_{j}$-isocline, where the selection gradient of the $j$ th strategy vanishes. A point on the $x_{j}$-isocline is uninvadable by mutants of $x_{j}$ if

$$
\partial_{11} r\left(x_{j}, \hat{E}_{1}\left(x_{1}, \ldots, x_{n}\right), \ldots, \hat{E}_{n}\left(x_{1}, \ldots, x_{n}\right)\right)<0
$$

(which is a sufficient condition; the same with $\leq$ is necessary). A point in $U_{1}$ where all $n$ isoclines intersect and therefore all selection gradients vanish is an $n$-morphic evolutionary singularity. If (11) is satisfied for all strategies $(j=1, \ldots, n)$ at the singularity, then the singularity is evolutionarily stable (sensu Maynard Smith 1982, p. 10). Since in degenerate cases equality may hold in (11) for some strategies of an evolutionarily stable singularity, we shall refer to a singularity where the strict inequalities are satisfied for each strategy as a generic evolutionarily stable singularity.

The selection gradients, the isoclines, the singularities, and their evolutionary stability are therefore determined by the trait values via the invasion fitness in equation (7), irrespectively of the embedding model in equation (8). Below we explore what information can be extracted from the invasion fitness and equation (7) alone for the adaptive dynamics of an $n$-morphic population.

The results obtained from equation (7) are biologically relevant only if the equilibrium of the population dynamics is admissible, i.e., the population densities are nonnegative and the equilibrium is asymptotically stable. As far as the environmental feedbacks are concerned, one can restrict the set $U_{1}$ of $n$-morphisms such that $\hat{E}_{1}\left(x_{1}, \ldots, x_{n}\right), \ldots, \hat{E}_{n}\left(x_{1}, \ldots, x_{n}\right)$ correspond to positive population densities for all $\left(x_{1}, \ldots, x_{n}\right) \in U_{1}$; often this will amount to requiring $\hat{E}_{i}\left(x_{1}, \ldots, x_{n}\right) \geq 0$ for all $i$, but there might be other constraints as well (for example, if $E_{1}$ is the total density of a biological species and $E_{2}$ is the density of individuals of the same species in a particular class, like juveniles, then one has to require $\hat{E}_{1} \geq \hat{E}_{2}$ ). Whether or not $N_{1}, \ldots, N_{n}$ and $Z_{1}, \ldots, Z_{k}$ are biologically possible and whether the equilibrium is asymptotically stable, however, depends on the embedding model in (8). Even though the results are of biological interest only if the equilibrium is admissible, the results themselves are not affected by the embedding model and hold for any $N_{1}, \ldots, N_{n}$ and $Z_{1}, \ldots, Z_{k}$. 


\subsection{Isoclines and singularities}

We first explore how the isoclines and singularities relate to the equilibria of the environmental feedback variables as functions of the trait values. Let

$$
\mathbf{A}=\left[\begin{array}{ccc}
\partial_{2} r\left(x_{1}, \hat{E}_{1}, \ldots, \hat{E}_{n}\right) & \ldots & \partial_{n+1} r\left(x_{1}, \hat{E}_{1}, \ldots, \hat{E}_{n}\right) \\
\vdots & \ddots & \vdots \\
\partial_{2} r\left(x_{n}, \hat{E}_{1}, \ldots, \hat{E}_{n}\right) & \ldots & \partial_{n+1} r\left(x_{n}, \hat{E}_{1}, \ldots, \hat{E}_{n}\right)
\end{array}\right]
$$

By implicit differentiation of equations (7) with respect to the trait value $x_{j}$, we obtain

$$
\mathbf{A}\left[\begin{array}{c}
\frac{\partial \hat{E}_{1}}{\partial x_{j}} \\
\vdots \\
\frac{\partial \hat{E}_{n}}{\partial x_{j}}
\end{array}\right]=-\partial_{1} r\left(x_{j}, \hat{E}_{1}, \ldots, \hat{E}_{n}\right) \mathbf{e}_{j}
$$

where $\mathbf{e}_{j}$ is the $j$ th unit vector of dimension $n$. Writing (13) for $j=1, \ldots, n$ yields $n$ matrix equations or a total of $n^{2}$ equations for the $n^{2}$ derivatives $\frac{\partial \hat{E}_{i}}{\partial x_{j}}(i, j=1, \ldots, n)$. Notice that A is independent of the choice of $j$.

We assume the following non-degeneracy condition on $\mathbf{A}$ :

(A4) The matrix $\mathbf{A}$ given in (12) and all its minor matrices $\mathbf{A}_{i j}$ obtained by deleting the $i$ th row and the $j$ th column of $\mathbf{A}$ are nonsingular when $\left(x_{1}, \ldots, x_{n}\right)$ is in some open set $U_{2} \subseteq \mathbb{X}^{n}$.

In sections 3.1-3.2, we restrict the analysis to the subset $U_{1} \cap U_{2}$ of the product trait space $\mathbb{X}^{n}$, so that assumptions (A2) and (A4) apply.

Equation (13) restricts how the equilibria of the environmental feedbacks vary with a trait value according to the following

Lemma 1. If one environmental feedback, $\hat{E}_{i}$, as a univariate function of the trait value $x_{j}$ with all other trait values fixed, has a critical point at $\left(x_{1}, \ldots, x_{n}\right)$, then this point is a critical point of all environmental feedbacks as univariate functions of $x_{j}$.

Proof. Given $\frac{\partial \hat{E}_{i}}{\partial x_{j}}=0$, we have from equation (13) that $\sum_{l=1}^{n} a_{k l} \frac{\partial \hat{E}_{l}}{\partial x_{j}}=0$ for all $k \neq j$ (where $a_{k l}$ is the $k, l$ element of $\mathbf{A}$ ), so that $\frac{\partial \hat{E}_{i}}{\partial x_{j}}=0$ implies $\sum_{l \neq i} a_{k l} \frac{\partial \hat{E}_{l}}{\partial x_{j}}=0$ for all $k \neq j$. This is a homogeneous linear system of equations for the $n-1$ derivatives $\frac{\partial \hat{E}_{l}}{\partial x_{j}}$, $l=1, \ldots, i-1, i+1, \ldots, n$. The matrix of this system is $\mathbf{A}_{j i}$, which is assumed to be nonsingular by (A4), so that the solution is the zero vector. Hence $\frac{\partial \hat{E}_{i}}{\partial x_{j}}=0$ implies $\frac{\partial \hat{E}_{l}}{\partial x_{j}}=0$ for all $l=1, \ldots, n$.

Proposition 1. If an environmental feedback $\hat{E}_{i}$, as a univariate function of $x_{j}$, has a critical point at $\left(x_{1}, \ldots, x_{n}\right)$, then this point is on the $x_{j}$-isocline of the saturated $n$ morphism. Conversely, at all points of the $x_{j}$-isocline each environmental feedback must have a critical point as a function of $x_{j}$. 
Proof. By Lemma 1, $\frac{\partial \hat{E}_{i}}{\partial x_{j}}=0$ implies $\frac{\partial \hat{E}_{l}}{\partial x_{j}}=0$ for all $l=1, \ldots, n$ such that the left hand side of $(13)$ is the zero vector. Therefore $\partial_{1} r\left(x_{j}, \hat{E}_{1}, \ldots, \hat{E}_{n}\right)=0$ and the point is on the $x_{j}$-isocline. Conversely, if the point $\left(x_{1}, \ldots, x_{n}\right)$ is on the $x_{j}$-isocline, then the right hand side of (13) is the zero vector, so that under (A4), $\frac{\partial \hat{E}_{i}}{\partial x_{j}}$ must be zero for all $i=1, \ldots, n$.

This result explains why, in Figure 1, the $\alpha_{1}$-isocline traces the points where the contour lines of both environmental feedback variables are horizontal (the feedbacks attain local minima or maxima when varying $\alpha_{1}$ ), and, analogously, the $\alpha_{2}$-isocline traces points where the contour lines of both environmental feedback variables are vertical (the feedbacks attain local minima or maxima when varying $\alpha_{2}$; recall that in the example of Figure 1, virulence is the trait value, i.e., $\left.x_{i}=\alpha_{i}\right)$.

Proposition 2. At an evolutionary singularity of a saturated n-morphism, each environmental feedback $\hat{E}_{i}(i=1, \ldots, n)$ has a critical point as a multivariate function of all trait values. Moreover, the Hessian matrix $\left[\frac{\partial^{2} \hat{E}_{i}}{\partial x_{j} \partial x_{k}}\right]$ at the $n$-morphic evolutionary singularity is a diagonal matrix for each environmental feedback $\hat{E}_{i}(i=1, \ldots, n)$.

Proof. At the evolutionary singularity all isoclines intersect, therefore by Proposition 1, each environmental feedback has a critical point as a function of all trait values. Differentiating equation (13) implicitly with respect to $x_{k}$ gives

$$
\frac{\partial \mathbf{A}}{\partial x_{k}}\left[\begin{array}{c}
\frac{\partial \hat{E}_{1}}{\partial x_{j}} \\
\vdots \\
\frac{\partial \hat{E}_{n}}{\partial x_{j}}
\end{array}\right]+\mathbf{A}\left[\begin{array}{c}
\frac{\partial^{2} \hat{E}_{1}}{\partial x_{j} \partial x_{k}} \\
\vdots \\
\frac{\partial^{2} \hat{E}_{n}}{\partial x_{j} \partial x_{k}}
\end{array}\right]=-b_{j k} \mathbf{e}_{j}
$$

with

$$
b_{j k}=\delta_{j k} \partial_{11} r\left(x_{j}, \hat{E}_{1}, \ldots, \hat{E}_{n}\right)+\sum_{l=1}^{n} \partial_{1, l+1} r\left(x_{j}, \hat{E}_{1}, \ldots, \hat{E}_{n}\right) \frac{\partial \hat{E}_{l}}{\partial x_{k}}
$$

where $\delta_{j k}$ is the Kronecker delta. At the intersection of the $x_{j}$ and $x_{k}$-isoclines, $\frac{\partial \hat{E}_{l}}{\partial x_{j}}=$ $\frac{\partial \hat{E}_{l}}{\partial x_{k}}=0$ holds for all $l=1, \ldots, n$ by Proposition 1 , and therefore (14) simplifies to

$$
\mathbf{A}\left[\begin{array}{c}
\frac{\partial^{2} \hat{E}_{1}}{\partial x_{j} \partial x_{k}} \\
\vdots \\
\frac{\partial^{2} \hat{E}_{n}}{\partial x_{j} \partial x_{k}}
\end{array}\right]=\left\{\begin{array}{cl}
-\partial_{11} r\left(x_{j}, \hat{E}_{1}, \ldots, \hat{E}_{n}\right) \mathbf{e}_{j} & \text { if } k=j \\
\mathbf{0} & \text { otherwise }
\end{array}\right.
$$

Whenever $k \neq j$, i.e., at the intersection of two different isoclines, it follows from assumption (A4) that $\frac{\partial^{2} \hat{E}_{i}}{\partial x_{j} \partial x_{k}}=0$ for $i=1, \ldots, n$. At the intersection of all isoclines the same holds for any choice of $k \neq j$, and therefore the Hessian matrix is diagonal.

In Figure 1, the dimorphic singularity is at a saddle point of each environmental feedback, and it can be seen from the contour lines of the feedbacks that the eigenvectors of their Hessians are the unit vectors so that the Hessians are diagonal matrices. 
Proposition 3. At an evolutionary singularity of a saturated n-morphism, the isoclines intersect at right angles such that the $x_{j}$-isocline $(j=1, \ldots, n)$ is tangent to a hyperplane perpendicular to the $x_{j}$ coordinate axis.

Proof. By Proposition 1, the $x_{j}$-isocline is implicitly determined by $\frac{\partial \hat{E}_{i}}{\partial x_{j}}=0$ (where $i$ is arbitrary). By implicit differentiation of this equation, we obtain $\sum_{k=1}^{n} \frac{\partial \hat{E}_{i}}{\partial x_{k} \partial x_{j}} d x_{k}=0$ to hold on the $x_{j}$-isocline, i.e., the normal vector of the $x_{j}$-isocline is $\left[\frac{\partial \hat{E}_{i}}{\partial x_{1} \partial x_{j}}, \ldots, \frac{\partial \hat{E}_{i}}{\partial x_{n} \partial x_{j}}\right]^{T}$. Since at the $n$-morphic singularity all mixed derivatives are zero by Proposition 2 , the normal vector of the $x_{j}$-isocline simplifies to $\frac{\partial^{2} \hat{E}_{i}}{\partial x_{j}^{2}} \mathbf{e}_{j}$.

This result explains why the $\alpha_{1}$-isocline is locally vertical and the $\alpha_{2}$ isocline is locally horizontal at the dimorphic singularity in Figure 1.

Proposition 4. If $n=2$ and the invasion fitness is uniformly monotonic in the environmental feedback variable $E_{i}$ for given $i$, then $\hat{E}_{i}\left(x_{1}, x_{2}\right)$, as a bivariate function of the trait values, has a saddle point at a generic evolutionarily stable dimorphic singularity; $\hat{E}_{i}\left(x_{1}, x_{2}\right)$ is maximized as a function of one trait value and minimized as a function of the other.

Proof. Take $k=j$ in equation (16) and use Cramer's rule to obtain

$$
\frac{\partial^{2} \hat{E}_{i}}{\partial x_{j}^{2}}=-\partial_{11} r\left(x_{j}, \hat{E}_{1}, \ldots, \hat{E}_{n}\right) \cdot(-1)^{i+j} \frac{\operatorname{det} \mathbf{A}_{j i}}{\operatorname{det} \mathbf{A}}
$$

for all $i, j$ at the singularity. In general, it is difficult to establish the signs of the determinants in this expression, but in the case of $n=2$, the minor matrices are simply elements of $\mathbf{A}$, such that $\operatorname{det} \mathbf{A}_{j i}=\partial_{i+1} r\left(x_{j}, \hat{E}_{1}, \hat{E}_{2}\right)$. If the invasion fitness is uniformly monotonic in the $i$ th environmental feedback variable, then $\operatorname{det} \mathbf{A}_{j i}$ has the same sign for $j=1$ and $j=2$. The second derivatives $\partial_{11} r\left(x_{1}, \hat{E}_{1}, \hat{E}_{2}\right)$ and $\partial_{11} r\left(x_{2}, \hat{E}_{1}, \hat{E}_{2}\right)$ characterize the uninvadability of the $x_{1^{-}}$and $x_{2}$-isoclines, respectively (cf. (11)), and they are both negative at a generic evolutionarily stable singularity. Since for given $i, \partial_{11} r\left(x_{j}, \hat{E}_{1}, \hat{E}_{2}\right)$ and $\operatorname{det} \mathbf{A}_{j i}$ do not change sign when changing $j$, but $(-1)^{i+j}$ does, the sign of (17) with $j=1$ is the opposite to its sign with $j=2$. Together with Proposition 1, this shows that $\hat{E}_{i}\left(x_{1}, x_{2}\right)$ is maximized as a function of one trait value and minimized as a function of the other, and thus has a saddle point at a generic evolutionarily stable dimorphic singularity.

In the example of section 2, the invasion fitness is uniformly increasing as a function of the density of susceptibles $(S)$ and uniformly decreasing as a function of predator density $(P)$. Accordingly, the dimorphic ESS is situated at a saddle point of both $\hat{S}$ and $\hat{P}$ (Figure 1). 


\subsection{Convergence stability}

The adaptive dynamics of $n$ coevolving scalar traits can be approximated by the canonical equation

$$
\begin{aligned}
\frac{d x_{1}}{d t}= & c_{1}\left(x_{1}, \ldots, x_{n}\right) \partial_{1} r\left(x_{1}, \hat{E}_{1}\left(x_{1}, \ldots, x_{n}\right), \ldots, \hat{E}_{n}\left(x_{1}, \ldots, x_{n}\right)\right) \\
& \vdots \\
\frac{d x_{n}}{d t} & =c_{n}\left(x_{1}, \ldots, x_{n}\right) \partial_{1} r\left(x_{n}, \hat{E}_{1}\left(x_{1}, \ldots, x_{n}\right), \ldots, \hat{E}_{n}\left(x_{1}, \ldots, x_{n}\right)\right)
\end{aligned}
$$

where the speed factors $c_{j}\left(x_{1}, \ldots, x_{n}\right)$ contain the frequency and size of mutations as well as the equilibrium population size and population structure of strategy $j$ in the $n$-morphism of strategies $x_{1}, \ldots, x_{n}$ (Dieckmann and Law 1996; Durinx et al. 2008). On the $x_{j}$-isocline, the right hand side of the $j$ th equation is zero, and the $n$-morphic singularity is a fixed point of the canonical equation. Below we discuss three stability concepts related to isoclines and singularities: isoclinic stability (Marrow et al. 1996), strong convergence stability (Marrow et al. 1996, Leimar 2009), and absolute stability (Matessi and Di Pascuale 1996, Leimar 2009).

We say that a point on the $x_{j}$-isocline is isoclinically stable if it is an attractor of the one-dimensional adaptive dynamics of $x_{j}$ assuming that all other trait values are fixed, i.e., if it is an attractor of (18) when $c_{j}>0$ and $c_{i} \equiv 0$ for all $i \neq j$.

Proposition 5. A point on the $x_{j}$-isocline of a saturated polymorphism is isoclinically stable if and only if it is uninvadable by mutants of $x_{j}$.

Proof. From (18), $\partial_{11} r\left(x_{j}, \hat{E}_{1}, \ldots, \hat{E}_{n}\right)+\sum_{i=1}^{n} \partial_{1, i+1} r\left(x_{j}, \hat{E}_{1}, \ldots, \hat{E}_{n}\right) \frac{\partial \hat{E}_{i}}{\partial x_{j}}<0$ is the sufficient condition for isoclinic stability (whereas the same with $\leq$ is necessary). By Proposition 1 , $\frac{\partial \hat{E}_{i}}{\partial x_{j}}=0$ for $i=1, \ldots, n$ on the $x_{j}$-isocline, and therefore the sufficient condition simplifies to $\partial_{11} r\left(x_{j}, \hat{E}_{1}, \ldots, \hat{E}_{n}\right)<0$ (necessary with $\leq$ ). This is the same as the condition for evolutionary stability in (11).

This explains why, in Figure 1, the horizontal (vertical) arrows point towards the $\alpha_{1^{-}}$ $\left(\alpha_{2^{-}}\right)$isocline where the isocline is uninvadable (thick continuous lines), but point away from the isocline where the isocline is invadable (dashed lines). Isoclinic stability is however neither necessary nor sufficient for an evolutionary singularity to be an attractor of the canonical equation when all strategies evolve (Marrow et al. 1996), and convergence to a fixed point of $(18)$ depends on the speed factors $c_{1}, \ldots, c_{n}$.

Following Leimar (2009), we say that the evolutionary singularity is strongly convergence stable if it is a locally asymptotically stable fixed point of (18) for any speed factors that are positive at the singularity and are continuously differentiable functions of the trait values. (Note that Leimar (2009) derives the conditions for strong convergence stability 
in case of a monomorphic population with an evolving trait vector, whereas we consider an $n$-morphic population where each strategy has a scalar trait. The difference is that in Leimar's model, a single mutation can cause a difference in all entries of the trait vector, whereas in our model, one mutation changes the trait value of only one strategy.)

Proposition 6. An evolutionary singularity of a saturated n-morphism is both evolutionarily stable and strongly convergence stable if $\partial_{11} r\left(x_{i}, \hat{E}_{1}, \ldots, \hat{E}_{n}\right)<0$ at the singularity for $i=1, \ldots, n$, whereas it is neither evolutionarily stable nor locally asymptotically stable for any positive speed factors if the (strict) opposite of any of these inequalities holds.

Proof. By Proposition 2, $\frac{\partial \hat{E}_{i}}{\partial x_{j}}=0$ for all $i$ and $j$ at the singularity. Hence the Jacobian of (18) simplifies to the diagonal matrix

$$
\left[\begin{array}{ccc}
c_{1} \partial_{11} r\left(x_{1}, \hat{E}_{1}, \ldots, \hat{E}_{n}\right) & \ldots & 0 \\
\vdots & \ddots & \vdots \\
0 & \ldots & c_{n} \partial_{n n} r\left(x_{n}, \hat{E}_{1}, \ldots, \hat{E}_{n}\right)
\end{array}\right]
$$

and therefore the fixed point is asymptotically stable if $\partial_{11} r\left(x_{i}, \hat{E}_{1}, \ldots, \hat{E}_{n}\right)<0$ for all $i$; and this condition coincides with the condition of evolutionary stability in (11).

Strong convergence stability assumes that the speed factors $c_{j}\left(x_{1}, \ldots, x_{n}\right)$ are continuously differentiable with respect to the trait values. We say that the singularity is absolutely stable (cf. Leimar 2009) if it is Lyapunov stable for any conceivable evolutionary trajectory, also when the speed factors are non-smooth functions of the trait values. This includes the most extreme path that is consistent with the selection gradients (see Matessi and Di Pascuale 1996). Contrary to Matessi and Di Pascuale (1996) and Leimar (2009), we consider Lyapunov stability rather than asymptotic stability because stasis is also a path consistent with the selection gradients. In Appendix B, we derive a sufficient condition for the absolute stability of an arbitrary (not necessarily saturated) polymorphic singularity, and then apply this condition to saturated polymorphisms.

Proposition 7. Every generic evolutionarily stable singularity of a saturated $n$-morphism is absolutely stable.

Proof. See Appendix B.

In Figure 1 it can easily be checked, by constructing the most extreme path, that the evolutionary trajectories cannot leave the neighbourhood of the dimorphic ESS.

\subsection{Limits to points on the diagonal}

In this section, we investigate the limit of the environmental feedbacks and of the selection gradients when two of the $n$ coexisting strategies approach the same trait value. In the case of $n=2$, this corresponds to the limit when the two trait values go to a point on 
the diagonal of the trait evolution plot (e.g. of Figure 1), $\left(x_{1}, x_{2}\right) \rightarrow\left(x_{0}, x_{0}\right)$. For $n>2$, we ease the presentation by assuming, without loss of generality, that the two strategies that approach the common value $x_{0}$ are the first two strategies, $x_{1}$ and $x_{2}$. For simplicity, we shall refer to the hyperplane $\left(x, x, x_{3}, \ldots, x_{n}\right)$ as "the diagonal" also in the case of $n>2$.

Let $x_{1}=x_{0}+\epsilon \xi_{1}$ and $x_{2}=x_{0}+\epsilon \xi_{2}$ with arbitrary $\xi_{1} \neq \xi_{2}$ and $\epsilon>0$, and let $\bar{E}_{1}(\epsilon), \ldots, \bar{E}_{n}(\epsilon)$ denote the equilibrium values of the environmental feedback variables obtained from the equations

$$
\begin{aligned}
r\left(x_{0}+\epsilon \xi_{1}, \bar{E}_{1}(\epsilon), \ldots, \bar{E}_{n}(\epsilon)\right) & =0 \\
r\left(x_{0}+\epsilon \xi_{2}, \bar{E}_{1}(\epsilon), \ldots, \bar{E}_{n}(\epsilon)\right) & =0 \\
r\left(x_{3}, \bar{E}_{1}(\epsilon), \ldots, \bar{E}_{n}(\epsilon)\right) & =0 \\
& \vdots \\
r\left(x_{n}, \bar{E}_{1}(\epsilon), \ldots, \bar{E}_{n}(\epsilon)\right) & =0
\end{aligned}
$$

We denote the equilibrium feedbacks with $\bar{E}_{i}$ rather than $\hat{E}_{i}$ because here we consider them as functions of $\epsilon$, with $\xi_{1}, \xi_{2}, x_{0}, x_{3}, \ldots, x_{n}$ suppressed in the notation. When $\epsilon=0$ (i.e., $x_{2}=x_{1}$ ), the model does not have isolated equilibria and the first two rows of $\mathbf{A}$ are identical so that $\mathbf{A}$ is singular, hence assumptions (A2) and (A4) do not hold. For the diagonal, we replace (A2) with the assumption

(A5) The limits $\bar{E}_{i}(0):=\lim _{\epsilon \rightarrow 0} \bar{E}_{i}(\epsilon)(i=1, \ldots, n)$ exist and are independent of the choice $\xi_{1} \neq \xi_{2}$. The limits $\bar{E}_{i}^{\prime}(0):=\lim _{\epsilon \rightarrow 0} \bar{E}_{i}^{\prime}(\epsilon)(i=1, \ldots, n)$ exist for all $\xi_{1} \neq \xi_{2}$ (these typically depend on the choice of $\xi_{1}, \xi_{2}$ ). If equations (7)-(8) have multiple solutions for the population dynamical equilibria, then the focal equilibrium, as a function of the trait values, extends continuously to the diagonal.

Proposition 8. As $\left(x_{1}, x_{2}, x_{3}, \ldots, x_{n}\right) \rightarrow\left(x_{0}, x_{0}, x_{3}, \ldots, x_{n}\right)$, the equilibrium values of the environmental feedback variables of a saturated n-morphism converge to the equilibrium feedbacks of an $(n-1)$-morphic population with strategies $\left(x_{0}, x_{3}, \ldots, x_{n}\right)$ where the selection gradient of the first strategy vanishes.

If $n=2$, then the equilibrium values of the environmental feedback variables converge to the equilibrium feedbacks of a monomorphic population if that has an evolutionary singularity at $x_{0}$.

(For $n>2$, the analogous result holds for every diagonal, i.e., for any pair of resident strategies $x_{i}$ and $x_{j}$ going to the same trait value $x_{0}$.)

Proof. From the difference of the first two equations of (20), we obtain

$$
\lim _{\epsilon \rightarrow 0} \frac{r\left(x_{0}+\epsilon \xi_{1}, \bar{E}_{1}(\epsilon), \ldots, \bar{E}_{n}(\epsilon)\right)-r\left(x_{0}+\epsilon \xi_{2}, \bar{E}_{1}(\epsilon), \ldots, \bar{E}_{n}(\epsilon)\right)}{\epsilon\left(\xi_{1}-\xi_{2}\right)}=\partial_{1} r\left(x_{0}, \bar{E}_{1}(0), \ldots, \bar{E}_{n}(0)\right)=0
$$


so that in the limit $\epsilon \rightarrow 0$, we can rewrite the system of equations as

$$
\begin{aligned}
\partial_{1} r\left(x_{0}, \bar{E}_{1}(0), \ldots, \bar{E}_{n}(0)\right) & =0 \\
r\left(x_{0}, \bar{E}_{1}(0), \ldots, \bar{E}_{n}(0)\right) & =0 \\
r\left(x_{3}, \bar{E}_{1}(0), \ldots, \bar{E}_{n}(0)\right) & =0 \\
& \vdots \\
r\left(x_{n}, \bar{E}_{1}(0), \ldots, \bar{E}_{n}(0)\right) & =0
\end{aligned}
$$

The same system of equations must hold if the point $\left(x_{0}, x_{3}, \ldots, x_{n}\right)$ is on the $x_{1}$-isocline of an $(n-1)$-morphic population or, in case of $n=2$, if $x_{0}$ is a monomorphic evolutionary singularity.

To interpret the above proposition, recall that it depends on the embedding model whether or not an $(n-1)$-morphic population is on its $x_{1}$-isocline (or a monomorphic population has a singularity at $x_{0}$ ). However, if the embedding model is such that $\left(x_{0}, x_{3}, \ldots, x_{n}\right)$ is in fact on the $x_{1}$-isocline (or $x_{0}$ is a monomorphic singularity), then the feedbacks of the $(n-1)$-morphic population with these traits are equal to the limits $\bar{E}_{i}(0)$.

For $n>2$, the case when $l>2$ strategies approach the same trait value can be treated analogously, and with higher $l$, this limit leads to increasingly degenerate isocline points or singularities. Consider the example $\left(x_{1}, x_{2}, x_{3}\right) \rightarrow\left(x_{0}, x_{0}, x_{0}\right)$ in a saturated trimorphism. Analogously to (20), we have

$$
\begin{aligned}
& r\left(x_{0}+\epsilon \xi_{1}, \overline{\mathbf{E}}(\epsilon)\right)=0 \\
& r\left(x_{0}+\epsilon \xi_{2}, \overline{\mathbf{E}}(\epsilon)\right)=0 \\
& r\left(x_{0}+\epsilon \xi_{3}, \overline{\mathbf{E}}(\epsilon)\right)=0
\end{aligned}
$$

where $\overline{\mathbf{E}}(\epsilon)$ abbreviates the arguments $\bar{E}_{1}(\epsilon), \bar{E}_{2}(\epsilon), \bar{E}_{3}(\epsilon)$. Taking the limit $\epsilon \rightarrow 0$, we obtain the first two equations of $(21)$ as well as

$$
\lim _{\epsilon \rightarrow 0} \frac{1}{\epsilon\left(\xi_{1}-\xi_{3}\right) / 2}\left[\frac{r\left(x_{0}+\epsilon \xi_{1}, \overline{\mathbf{E}}(\epsilon)\right)-r\left(x_{0}+\epsilon \xi_{2}, \overline{\mathbf{E}}(\epsilon)\right)}{\epsilon\left(\xi_{1}-\xi_{2}\right)}-\frac{r\left(x_{0}+\epsilon \xi_{2}, \overline{\mathbf{E}}(\epsilon)\right)-r\left(x_{0}+\epsilon \xi_{3}, \overline{\mathbf{E}}(\epsilon)\right)}{\epsilon\left(\xi_{2}-\xi_{3}\right)}\right]=\partial_{11} r\left(x_{0}, \overline{\mathbf{E}}(0)\right)=0
$$

The equilibrium values of the feedback variables therefore converge to the equilibrium feedbacks of a monomorphic population that has an evolutionary singularity at $x_{0}$ at the bifurcation point between being invadable and uninvadable (i.e., evolutionarily stable).

Proposition 9. The point $\left(x_{1}, \ldots, x_{n}\right)$ is on the $x_{j}$-isocline of the saturated $n$-morphic population if and only if at this point each environmental feedback variable has the same equilibrium value as its limit to the point $\left(x_{1}, \ldots, x_{l-1}, x_{j}, x_{l+1}, \ldots, x_{n}\right)$ for all $l \neq j$.

In the case of $n=2$, the point $\left(x_{1}, x_{2}\right)$ is on the $x_{1}$-isocline if and only if at this point each environmental feedback variable has the same equilibrium value as its limit to the point $\left(x_{1}, x_{1}\right)$. Likewise, the point $\left(x_{1}, x_{2}\right)$ is on the $x_{2}$-isocline if and only if at this point each environmental feedback variable has the same equilibrium value as its limit to the point $\left(x_{2}, x_{2}\right)$. 
Proof. If the point $\left(x_{1}, \ldots, x_{n}\right)$ is on the $x_{1}$-isocline, then $\partial_{1} r\left(x_{1}, E_{1}, \ldots, E_{n}\right)=0$ must hold together with equations (7). These are the same $n+1$ equations as the $n$ equations in (21) written for $x_{0}=x_{1}$ together with $r\left(x_{2}, E_{1}, \ldots, E_{n}\right)=0$. Hence on the $x_{1}$-isocline, $\hat{E}_{i}\left(x_{1}, \ldots, x_{n}\right)=\bar{E}_{i}(0)$. An analogous result holds for every diagonal (e.g. $\left(x_{1}, \ldots, x_{j-1}, x_{1}, x_{j+1}, \ldots, x_{n}\right)$ instead of $\left.\left(x_{1}, x_{1}, x_{3}, \ldots, x_{n}\right)\right)$ and for every isocline.

This result underlies that in Figure 1, the $\alpha_{1}$-isocline traces points where each feedback has the same value as its limit to the diagonal at the same $\alpha_{1}$-coordinate, whereas the $\alpha_{2}$-isocline traces points where each feedback has the same value as its limit to the diagonal at the same $\alpha_{2}$-coordinate (see the white guide lines in the bottom panels).

In the remainder of this section, we investigate the points where the isoclines of a saturated polymorphism intersect the diagonal (see a summary for the simplest case $n=2$ at the end of this section). Let

$$
X^{c}=\left\{x: \partial_{11} r\left(x, \bar{E}_{1}(0), \ldots, \bar{E}_{n}(0)\right)=0\right\}
$$

Generically, $X^{c}$ consists of isolated points. Note that for $n>2, X^{c}$ depends on the choice of $x_{3}, \ldots, x_{n}$, and when necessary, we emphasise this by writing $X^{c}\left(x_{3}, \ldots, x_{n}\right)$. Suppose that the embedding model is such that the point $\left(x_{0}, x_{3}, \ldots, x_{n}\right)$ is on the $x_{1}$-isocline of the $(n-1)$-morphic population; by Proposition 8 , the equilibrium of the environmental feedbacks in this this $(n-1)$-morphic population is given by $\bar{E}_{1}(0), \ldots, \bar{E}_{n}(0)$. If $x_{0} \in X^{c}$, then $\left(x_{0}, x_{3}, \ldots, x_{n}\right)$ is at a bifurcation point between being invadable and uninvadable by mutants of the first strategy. In particular, if $n=2$ and the embedding model is such that $x_{0} \in X^{c}$ is a monomorphic singularity, then $x_{0}$ is at a bifurcation point between a generic ESS and an invadable singularity (such as an evolutionary branching point). The following two propositions link the elements of $X^{c}$ to the environmental feedback variables and to the isoclines of the $n$-morphic population under the non-degeneracy condition given in assumption (A8) in Appendix C.

Proposition 10. The points $\left(x_{0}, x_{0}, x_{3}, \ldots, x_{n}\right)$ of the diagonal with $x_{0} \in X^{c}\left(x_{3}, \ldots, x_{n}\right)$ have the following properties:

(i) The $x_{1}$ - and $x_{2}$-isoclines of the saturated $n$-morphic population intersect the diagonal exactly at these points;

(ii) The limiting value of each equilibrium environmental feedback attains a critical point as a function of $x_{1}$ and $x_{2}$ at exactly these points.

(For $n>2$, the analogous result holds for every diagonal.)

Proof. See Appendix C.

Corollary. At the point(s) $\left(x_{0}, \ldots, x_{0}\right)$ with $x_{0} \in X^{c}\left(x_{0}, \ldots, x_{0}\right)$ all isoclines of the $n$-morphic population intersect and the limiting value of each equilibrium environmental feedback variable attains a critical point as a function of all trait values, provided that the limits of $\hat{E}_{i}\left(x_{1}, \ldots, x_{n}\right)$ and its first derivatives exist for all $i$ when $\left(x_{1}, \ldots, x_{n}\right) \rightarrow\left(x_{0}, \ldots, x_{0}\right)$. 
Proposition 11. The $x_{1}$ - and $x_{2}$-isoclines of the n-morphic population have common points with the $x_{1}$-isocline of the $(n-1)$-morphic population exactly at the points where the latter bifurcates between being invadable and uninvadable by mutants of the first strategy.

In the case of $n=2$, the $x_{1}$ - and $x_{2}$-isoclines connect to the monomorphic singularity if and only if the monomorphic singularity is at a bifurcation point between a generic ESS and an invadable singularity.

(For $n>2$, the analogous result holds for every pair of $n$-morphic isoclines.)

Proof. This follows directly from the fact that the $x_{1}$-isocline of the $(n-1)$-morphic population is embedded in the diagonal $\left(x, x, x_{3}, \ldots, x_{n}\right)$ and, by part (i) of Proposition 10 , the $x_{1}$ and $x_{2}$-isoclines of the $n$-morphic population intersect the diagonal at the points $\left\{\left(x, x, x_{3}, \ldots, x_{n}\right) \mid x \in X^{c}\left(x_{3}, \ldots, x_{n}\right)\right\}$.

For dimorphisms, Proposition 11 can be deduced from the Appendix of Geritz et al. (1999), which holds true also in the unsaturated case. Moreover, this general result for dimorphisms can be extended to arbitrary $n$-morphisms by fixing strategies $x_{3}, \ldots, x_{n}$. The above short proof of Proposition 11, however, assumes that the $n$-morphism is saturated.

Proposition 12. In the case of saturated dimorphisms $(n=2)$, the vector of selection gradients is asymptotically perpendicular to the diagonal as $\left(x_{1}, x_{2}\right) \rightarrow\left(x_{0}, x_{0}\right)$, and it points towards (away from) the diagonal if $\partial_{11} r\left(x_{0}, \bar{E}_{1}(0), \ldots, \bar{E}_{n}(0)\right)$ is negative (positive).

Proof. See Appendix C.

For $n=2$, the results in this section imply the following relationship between the adaptive dynamics of monomorphic and dimorphic populations. The diagonal of the trait evolution plot is divided into segments separated by the points $\left(x_{0}, x_{0}\right)$ with $x_{0} \in X^{c}$, at which points the isoclines intersect the diagonal and the (limits of the) environmental feedbacks have critical points. Within each segment, the monomorphic population may have either an ESS or an invadable singularity (such as an evolutionary branching point) depending on whether the vector of the dimorphic selection gradients points towards the diagonal or away from the diagonal (cf. Geritz et al. 1998). The location of the monomorphic singularity depends on the embedding model, but given the location, its invadability does not. In the example of Figure 1, $X^{c}$ contains the two elements 0.932 and 3.044. A monomorphic singularity at $x_{0}$ is invadable if $x_{0}$ is in the interval $(0.932,3.044)$ and evolutionarily stable if it is outside [0.932, 3.044].

\section{Embedding models and invasion boundaries}

In this section, we study how the connections between the adaptive dynamics of saturated $n$-morphisms and the invasibility of $(n-1)$-morphisms depend on the embedding model in equations (8). For $n=2$, this amounts to the relationship between the dimorphic selection gradients (i.e., the constant "backdrop" in the right-hand panels of Figure 2) 
and the pairwise invasibility plot of a monomorphic population.

The invasion fitness of a mutant strategy $x_{m}$ in the $(n-1)$-morphic resident population of strategies $x_{1}, \ldots, x_{m-1}, x_{m+1}, \ldots, x_{n}$ changes sign when, in the population dynamics of the $n$-morphism, the boundary equilibrium $\left(N_{1}, \ldots, N_{m-1}, 0, N_{m+1}, \ldots, N_{n}\right)$ undergoes a transcritical bifurcation. The manifold of this transcritical bifurcation is an $(n-1)$ dimensional manifold in the $n$-dimensional product trait space, which we refer to as the $x_{m}$-invasion boundary. In the case of $n=2$, the $x_{2}$-invasion boundary is the line of the pairwise invasibility plot that separates "+" areas of invasion from "-" areas of noninvasion in the left panels of Figure 2 . The $x_{1}$-invasion boundary is the mirror image of the $x_{2}$-invasion boundary on the diagonal $x_{2}=x_{1}$; in Figure 2, it appears in the right panels delineating the area of coexistence together with the $x_{2}$-invasion boundary. The $x_{m}$-invasion boundary is implicitly determined by

$$
\begin{array}{rc}
F_{1}\left(x_{1}, \ldots, x_{n}, \hat{E}_{1}(\mathbf{x}), \ldots, \hat{E}_{n}(\mathbf{x}), N_{1}, \ldots, N_{m-1}, 0, N_{m+1}, \ldots, N_{n}, Z_{1}, \ldots, Z_{k}\right) & =0 \\
F_{n+k}\left(x_{1}, \ldots, x_{n}, \hat{E}_{1}(\mathbf{x}), \ldots, \hat{E}_{n}(\mathbf{x}), N_{1}, \ldots, N_{m-1}, 0, N_{m+1}, \ldots, N_{n}, Z_{1}, \ldots, Z_{k}\right) & =0
\end{array}
$$

where $\hat{E}_{i}(\mathbf{x})=\hat{E}_{i}\left(x_{1}, \ldots, x_{n}\right)$ for $i=1, \ldots, n$ are determined by equation $(7)$.

\subsection{Connection between isoclines and invasion boundaries}

Geritz et al. (1999, see their Appendix) have shown that the isoclines of dimorphic selection gradients connect to the invasion boundaries of the pairwise invasibility plot at specific points. Here we generalize their results to the isoclines of saturated $n$-morphisms. We conjecture that Propositions 13 and 14 are valid also for unsaturated $n$-morphisms, but proving them for the unsaturated case is beyond the present paper.

Lemma 2. If the point $\left(x_{1}, \ldots, x_{m}, \ldots, x_{n}\right)$ is on the $x_{m}$-invasion boundary and $\bar{x}_{m}$ is such that $\hat{E}_{i}\left(x_{1}, \ldots, \bar{x}_{m}, \ldots, x_{n}\right)=\hat{E}_{i}\left(x_{1}, \ldots, x_{m}, \ldots, x_{n}\right)$ for $i=1, \ldots, n$, then the point $\left(x_{1}, \ldots, \bar{x}_{m}, \ldots, x_{n}\right)$ is also on the $x_{m}$-invasion boundary.

Proof. By assumption (A3), the functions $F_{i}$ in equations (23) do not depend on the value of their $m$ th argument. Hence $x_{m}$ enters equations (23) only via the environmental feedbacks $\hat{E}_{1}, \ldots, \hat{E}_{n}$, and if these are the same at $\left(x_{1}, \ldots, \bar{x}_{m}, \ldots, x_{n}\right)$ as at $\left(x_{1}, \ldots, x_{m}, \ldots, x_{n}\right)$, then $\left(x_{1}, \ldots, \bar{x}_{m}, \ldots, x_{n}\right)$ also satisfies $(23)$.

For $n=2$, this lemma implies that if a point is on the $x_{2}$-invasion boundary, then every point with the same $x_{1}$-coordinate and with the same values of $\hat{E}_{1}$ and $\hat{E}_{2}$ (cf. Figure 1) are also on the invasion boundary. Hence the equilibrium values of the environmental feedback variables in dimorphic populations constrain the possible shapes of pairwise invasibility plots. 
In the next two propositions, we first consider the intersection of an invasion boundary with the isocline of a different strategy, and then the intersection of the $x_{m}$-invasion boundary with the $x_{m}$-isocline.

Proposition 13. If the point $\left(x_{1}, \ldots, x_{m}, \ldots, x_{n}\right)$ is both on the $x_{j}$-isocline and on the $x_{m}$-invasion boundary with $j \neq m$, then the $(n-1)$-morphic population obtained by deleting strategy $x_{m}$ is also on its $x_{j}$-isocline. Conversely, if the $(n-1)$-morphic population obtained by deleting $x_{m}$ is on the $x_{j}$-isocline of the $(n-1)$-morphism and the point $\left(x_{1}, \ldots, x_{m}, \ldots, x_{n}\right)$ is on the $x_{j}$-isocline of the $n$-morphism, then $\left(x_{1}, \ldots, x_{m}, \ldots, x_{n}\right)$ is on the $x_{m}$-invasion boundary.

In the case of $n=2$, if a point $\left(x_{1}, x_{2}\right)$ is on the $x_{1}$-isocline and also on the $x_{2}$-invasion boundary, then $x_{1}$ is a monomorphic singularity; and conversely, if $x_{1}$ is a monomorphic singularity and $\left(x_{1}, x_{2}\right)$ is on the $x_{1}$-isocline, then it is also on the $x_{2}$-invasion boundary. Analogously, if $\left(x_{1}, x_{2}\right)$ is on the $x_{2}$-isocline and also on the $x_{1}$-invasion boundary, then $x_{2}$ is a monomorphic singularity; and conversely, if $x_{2}$ is a monomorphic singularity and $\left(x_{1}, x_{2}\right)$ is on the $x_{2}$-isocline, then it is also on the $x_{1}$-invasion boundary.

Proof. Consider the case $n=2$. If the point $\left(x_{1}, x_{2}\right)$ is on the $x_{1}$-isocline, then, by Proposition 9, $\hat{E}_{i}\left(x_{1}, x_{2}\right)$ equals its limit to $\left(x_{1}, x_{1}\right)$ for $i=1,2$. If $\left(x_{1}, x_{2}\right)$ is also on the $x_{2}$-invasion boundary, then, by Lemma $2,\left(x_{1}, x_{1}\right)$ must be on the $x_{2}$-invasion boundary, i.e., must be a point where the $x_{2}$-invasion boundary intersects the diagonal $x_{2}=x_{1}$. Hence $x_{1}$ is a monomorphic singularity. Conversely, if $x_{1}$ is a monomorphic singularity, then the point $\left(x_{1}, x_{1}\right)$ is on the $x_{2}$-invasion boundary. By Lemma 2 , every point $\left(x_{1}, x_{2}\right)$ that has the same equilibrium values of the environmental feedback variables as their limiting values at $\left(x_{1}, x_{1}\right)$ are also on the $x_{2}$-invasion boundary. By Proposition 9 , these points are on the $x_{1}$-isocline. The analogous statements for the $x_{2}$-isocline and $x_{1}$-invasion boundary follow from symmetry. For $n>2$, see Appendix D.

Proposition 14. At all points where the $x_{m}$-isocline intersects the $x_{m}$-invasion boundary, the $x_{m}$-invasion boundary is tangent to an $(n-1)$-dimensional hyperplane parallel to the $x_{m}$-axis.

Proof. See Appendix D.

Note that that Proposition 13 needs assumption (A5) but Proposition 14 does not. In the case of $n=2$, Proposition 13 means that whenever the $x_{1}$-isocline intersects the $x_{2}$-invasion boundary, there is a monomorphic singularity on the diagonal at the same $x_{1}$-coordinate; and whenever the $x_{2}$-isocline intersects the $x_{1}$-invasion boundary, there is a monomorphic singularity on the diagonal at the same $x_{2}$-coordinate. Proposition 14 implies that the $x_{2}$-isocline connects to the leftmost and to the rightmost points of the $x_{2}$-invasion boundary (i.e. to points where the $x_{2}$-invasion boundary is locally vertical), whereas the $x_{1}$-isocline connects to the minima and to the maxima of the $x_{1}$-invasion boundary (i.e., where the $x_{1}$-invasion boundary is locally horizontal). These connection points can readily be observed in Figure 2 (see also Geritz et al. 1999). 


\subsection{Embedding models and pairwise invasibility plots for $n=2$}

In this section, we show how certain elements of the embedding model can be chosen such that we obtain a particular segment of the invasion boundary according to will. This construction method demonstrates how the invasion boundaries can change while the $n$ morphic adaptive dynamics remain otherwise the same. For simplicity, we restrict this analysis to $n=2$, and construct parts of the $x_{2}$-invasion boundary, i.e., of the borderline between "+" and "-" areas of the pairwise invasibility plot, over a constant "backdrop" of the dimorphic selection gradients (cf. Figure 2).

Suppose that the embedding model contains a function $h$ the shape of which we may choose. $h$ could stand for a density-dependent demographic rate, such as the densitydependent birth rate of the host in our motivating example (function $B$ in equation (2)). For the sake of simplicity, we assume that $h$ is univariate and occurs only in $F_{1}$, such that the embedding model is as given in (8), except that the first equation is replaced with

$$
\tilde{F}_{1}\left(x_{1}, x_{2}, \hat{E}_{1}\left(x_{1}, x_{2}\right), \hat{E}_{2}\left(x_{1}, x_{2}\right), N_{1}, N_{2}, Z_{1}, \ldots, Z_{k}, h(f)\right)=0
$$

where $f=f\left(x_{1}, x_{2}, \hat{E}_{1}\left(x_{1}, x_{2}\right), \hat{E}_{2}\left(x_{1}, x_{2}\right), N_{1}, N_{2}, Z_{1}, \ldots, Z_{k}\right)$ such that $h$ may depend on an arbitrary combination of the demographic variables (in our motivating example, the birth rate depends on the sum of susceptible and infected densities, i.e., on the sum of one of the feedback variables and the two resident population densities). We make two assumptions about $\tilde{F}_{1}$ and $f$ :

(A6) $\tilde{F}_{1}$ is a $C^{1}$-diffeomorphism as a function of $h$ for all values of its other arguments.

(A7) If $N_{j}=0$, then $f$ is constant with respect to $x_{j}(j=1,2)$.

In many simple models $\tilde{F}_{1}$ is linear in $h$, so that (A6) holds trivially (for example, equation (2a) is linear in $B)$. (A7) is analogous to assumption (A3) and expresses the biological fact that if a strategy is not present, its trait value is irrelevant. We use (A7) in Appendix E. It is important that the invasion fitness does not contain $h$, so that changing $h$ does not affect the selection gradients of dimorphic populations.

From $(23)$, a point $\left(x_{1}, x_{2}\right)$ is on the $x_{2}$-invasion boundary if

$$
\begin{aligned}
& \tilde{F}_{1}\left(x_{1}, x_{2}, \hat{E}_{1}\left(x_{1}, x_{2}\right), \hat{E}_{2}\left(x_{1}, x_{2}\right), N_{1}, 0, Z_{1}, \ldots, Z_{k}, h(f)\right)=0 \\
& F_{2}\left(x_{1}, x_{2}, \hat{E}_{1}\left(x_{1}, x_{2}\right), \hat{E}_{2}\left(x_{1}, x_{2}\right), N_{1}, 0, Z_{1}, \ldots, Z_{k}\right)=0 \\
& \quad \vdots \\
& F_{k+2}\left(x_{1}, x_{2}, \hat{E}_{1}\left(x_{1}, x_{2}\right), \hat{E}_{2}\left(x_{1}, x_{2}\right), N_{1}, 0, Z_{1}, \ldots, Z_{k}\right)=0
\end{aligned}
$$

Let $N_{1}$ and $Z_{1}, \ldots, Z_{k}$ take values that satisfy the last $k+1$ equations in (24), and let $\hat{f}\left(x_{1}, x_{2}\right)$ denote the value of $f$ at these arguments and $N_{2}=0$. The point $\left(x_{1}, x_{2}\right)$ is on the invasion boundary if $h$ is chosen such that $h\left(\hat{f}\left(x_{1}, x_{2}\right)\right)=\hat{h}_{0}\left(x_{1}, x_{2}\right)$, where the value $\hat{h}_{0}\left(x_{1}, x_{2}\right)$ is the solution of

$$
\tilde{F}_{1}\left(x_{1}, x_{2}, \hat{E}_{1}\left(x_{1}, x_{2}\right), \hat{E}_{2}\left(x_{1}, x_{2}\right), N_{1}, 0, Z_{1}, \ldots, Z_{k}, \hat{h}_{0}\left(x_{1}, x_{2}\right)\right)=0
$$


Assumption (A6) guarantees that $\hat{h}_{0}\left(x_{1}, x_{2}\right)$ exists and is unique. One can make the invasion boundary follow a parameterised curve $\left(x_{1}(p), x_{2}(p)\right)$ by choosing $h$ such that $h\left(\hat{f}\left(x_{1}(p), x_{2}(p)\right)\right)=\hat{h}_{0}\left(x_{1}(p), x_{2}(p)\right)$, but this is possible only as long as $p \mapsto \hat{f}\left(x_{1}(p), x_{2}(p)\right)$ is an injection.

In Appendix E, we show that the invasion boundary is tangent to a line with slope $\kappa$ at the point $\left(x_{1}, x_{2}\right)$ if $h$ is chosen according to

$$
h(f)=\hat{h}_{0}\left(x_{1}, x_{2}\right)+\hat{h}_{1}\left(x_{1}, x_{2} ; \kappa\right)\left(f-\hat{f}\left(x_{1}, x_{2}\right)\right)+O\left(\left(f-\hat{f}\left(x_{1}, x_{2}\right)\right)^{2}\right)
$$

with $\hat{h}_{1}\left(x_{1}, x_{2} ; \kappa\right)$ given by equation (47) in Appendix E.

This construction method gives considerable freedom for obtaining pairwise invasibility plots with certain desired local properties. Three important caveats must however be made. First, by Lemma 2 in section 4.1, certain points of the invasion boundary are linked to each other via the environmental feedback variables, so that forcing the invasion boundary to a given point can create other invasion boundary points as well. Second, it remains to be seen whether the population dynamical equilibrium of the dimorphism $\left(x_{1}, x_{2}\right)$ with $h(f)$ given in $(25)$ is admissible. Third, on which side of the invasion boundary the mutants can or cannot invade (which is the "+" vs " -" side of the invasion boundary on the pairwise invasibility plot) is determined by the invasion fitness, not the embedding model. If an invasion boundary goes through the point $\left(x_{1}, x_{2}\right)$, then a mutant strategy $x_{2}+\delta$ can invade the monomorphic resident population of $x_{1}$ if the sign of $\partial_{1} r\left(x_{2}, \hat{E}_{1}\left(x_{1}, x_{2}\right), \hat{E}_{2}\left(x_{1}, x_{2}\right)\right)$ is the same as the sign of $\delta$.

In the remainder of this section, we show how this construction method can be used to obtain a monomorphic singularity of a desired type. Generic monomorphic singularities occur where the invasion boundary intersects the diagonal. The stability properties of a monomorphic singularity at $x_{0}$ are characterized by the second derivatives

$$
C_{00}=\left.\frac{\partial^{2} s_{x}(y)}{\partial y^{2}}\right|_{y=x=x_{0}} \text { and } C_{11}=\left.\frac{\partial^{2} s_{x}(y)}{\partial x^{2}}\right|_{y=x=x_{0}}
$$

where $s_{x}(y)$ is the invasion fitness of mutant strategy $y$ in the resident population of $x$, i.e., $s_{x}(y)=r\left(y, E_{1}, E_{2}\right)$ with $E_{1}$ and $E_{2}$ assuming their equilibrium values in the monomorphic population of strategy $x$ (Geritz et al. 1998). The eight generic types of monomorphic singularities are distinguished by the signs of $C_{00}, C_{11}$, and $\left|C_{00}\right|-\left|C_{11}\right|$ (see Figure 2 of Geritz et al. 1998). A simple calculation shows that the slope of the invasion boundary at the monomorphic singularity is $\kappa=C_{11} / C_{00}$.

Taking the limit $\left(x_{1}, x_{2}\right) \rightarrow\left(x_{0}, x_{0}\right)$ in $(25)$, we can construct $h$ such that the invasion boundary intersects the diagonal at an arbitrarily chosen point $\left(x_{0}, x_{0}\right)$. This means that we can force a monomorphic singularity to exist at $x_{0}$. Recall that $C_{00}=$ $\partial_{11} r\left(x_{0}, \bar{E}_{1}(0), \bar{E}_{2}(0)\right)$ is determined independently of the embedding model (cf. Proposition 12) so that the choice of $h$ will not influence it, and $C_{00}$ is zero when $x_{0} \in X^{c}$. By 
varying $\kappa$, however, we can achieve any value of $C_{11}$, and therefore we can construct any of the four types of monomorphic singularities admitted by the sign of $C_{00}$ (see Appendix $\mathrm{E}$ on the case of $C_{00}=0$ ). If $X^{c}$ is nonempty (which is easiest seen from whether the dimorphic isoclines intersect the diagonal, see Proposition 10), then generically the diagonal has segments with negative and with positive $C_{00}$. In this case, one can construct $h$ to obtain an arbitrary monomorphic singularity: first choose $x_{0}$ such that $C_{00}$ has the appropriate sign, and then choose $\kappa$ to obtain the desired singularity. Once again, this is up to the admissibility of the population dynamical equilibrium.

\section{Discussion}

We studied the adaptive dynamics of coexisting strategies assuming that the invasion fitness depends on a finite number $n$ of environmental feedback variables and the maximum number of strategies that can coexist in equilibrium, given by $n$, are already present. In the saturated polymorphism of strategies $x_{1}, \ldots, x_{n}$, the equilibrium values assumed by the environmental feedback variables, $\hat{E}_{1}\left(x_{1}, \ldots, x_{n}\right), \ldots, \hat{E}_{n}\left(x_{1}, \ldots, x_{n}\right)$, are determined from the fact that residents are selectively neutral in their self-generated environment, so that the invasion fitness of each resident strategy is zero (see equation (7)). The selection gradients, their isoclines, and the evolutionary singularities depend only on the environmental feedback variables, and are thus independent of all model details that do not feature in the invasion fitness.

We can therefore separate the equations of the ecological model into two sets. The first set consists of the $n$ equations that directly describe the dynamics of the population densities (or, in case of structured populations, the norms of the population density vectors) of the coexisting strategies. These equations contain the expression of the invasion fitness as a function of the trait value and of the environmental feedback variables (equations (1) in our motivating example of section 2). The second set of equations, to which we refer to as the embedding model, consists of all other equations of the ecological model, including the equations for the dynamics of the environmental feedback variables (equations (2) in the example of section 2). The selection gradients, isoclines, and evolutionary singularities of saturated polymorphisms are independent of the embedding model. In other words, all information contained in Figure 1 derives from the invasion fitness alone.

The embedding model, however, affects the positivity and stability of the population dynamical equilibrium (see Appendix A on the extension to non-equilibrium population dynamics). While the invasion fitness alone determines the position and the adaptive dynamic stability of the $n$-morphic singularities, it remains to be seen whether these singularities are admissible in the sense that they correspond to biologically feasible and ecologically stable systems (see Figure 2). 
The dimensionality of the environmental feedback may be infinite. This occurs also in very simple models, such as the trait-dependent Lotka-Volterra competition model (Metz et al. 1996a; Kisdi 1999), where the feedback environment is given by a function rather than by scalars. However, since models tend to investigate simple ecosystems, if the dimensionality of the feedback is finite, then it is often low. Dimorphisms are saturated in a number of models (see examples in the Introduction and in section 2), so that evolutionary branching in a monomorphic population, studied by most models, leads directly to a saturated system. In reality, the number of environmental feedback variables is likely high, but many of the feedbacks may have only a weak effect on the invasion fitness. These "weak" feedbacks can facilitate coexistence in only a narrow part of the product trait space (cf. Meszéna et al. 2006), and outside this part the adaptive dynamics of the system is well approximated by a model omitting the "weak" feedbacks, leaving only relatively few.

The propositions of section 3 characterize the adaptive dynamics of saturated polymorphisms. Some of these results are analogous to optimization models (which are saturated with $n=1$; see Metz et al. 2008). Importantly, the conditions for evolutionary stability and convergence stability coincide in the $n$-dimensional adaptive dynamics of saturated polymorphisms (Propositions 6 and 7) as they do in optimization models (Meszéna et al. 2001; Metz et al. 2008). In unsaturated polymorphisms, convergence stability may depend on the speed factors of the canonical equation (see equation 18), and there are several stability concepts that differ in the assumptions about the speed factors (Leimar 2009). In Appendix B, we give a new sufficient condition for the absolute stability of an arbitrary (not necessarily saturated) $k$-morphic singularity. This has previously been available only for $k=2$ (Matessi and Di Pascuale 1996; Kisdi 2006). This sufficient condition is always satisfied at evolutionarily stable singularities of saturated polymorphisms $(k=n$; see Proposition 7).

The adaptive dynamics of coevolving strategies may exhibit limit cycles (Khibnik and Kondrashov 1997), and a $k$-morphic evolutionary singularity (which is a fixed point of the canonical equation for $k$ strategies) can undergo a Hopf bifurcation (see e.g. Marrow et al. 1996; Gavrilets 1997; Dercole et al. 2003, 2010). At the singularities of saturated polymorphisms, however, the Jacobian evaluates to a diagonal matrix (see (19)), so that a Hopf bifurcation cannot occur. This again resembles the adaptive dynamics of optimization models, which have a strict Lyapunov function and therefore converge to the optimal strategy.

In optimization models, a (locally) optimal strategy corresponds to an extremum of the equilibrium value of the single environmental feedback, $\hat{E}(x)$, as a function of the resident strategy $x$, and if the invasion fitness $r(x, E)$ increases in $E$, then this extremum is a minimum. This has been shown by Mylius and Diekmann (1995) and Metz et al. (1996b, 2008), and it also follows directly from our equation (17) with $n=1$. In saturated $n$-morphisms, the evolutionarily stable singularities still correspond to critical points of 
each environmental feedback (Proposition 2), but these critical points are generally not extrema when $n \geq 2$. In particular, in the case of $n=2$, the evolutionarily stable dimorphic singularities are saddle points of $\hat{E}_{1}\left(x_{1}, x_{2}\right)$ and $\hat{E}_{2}\left(x_{1}, x_{2}\right)$, such that an environmental feedback is maximized as a function of one trait value and minimized as a function of the other (Proposition 4).

To explain this last result heuristically, suppose that the invasion fitness is an increasing function of both $E_{1}$ and $E_{2}$, and focus attention on the neighbourhood of a dimorphic singularity $\left(x_{1}, x_{2}\right)$. Comparing the singular dimorphism with the dimorphism $\left(x_{1}+d x_{1}, x_{2}\right)$, if we have $\hat{E}_{1}\left(x_{1}+d x_{1}, x_{2}\right)>\hat{E}_{1}\left(x_{1}, x_{2}\right)$, then we must have $\hat{E}_{2}\left(x_{1}+d x_{1}, x_{2}\right)<\hat{E}_{2}\left(x_{1}, x_{2}\right)$. This is because in both dimorphisms, the resident strategy $x_{2}$ must have zero invasion fitness, so that an increase in $\hat{E}_{1}$ must be compensated by a decrease in $\hat{E}_{2}$. Hence moving horizontally in the $\left(x_{1}, x_{2}\right)$-plane, the two feedbacks change in opposite directions. By an analogous argument, the same holds also vertically. (Note that Figure 1 is different because in the model of section 2, the invasion fitness increases in $S$ but decreases in $P$.) Next, recall that in order to coexist, $x_{1}$ and $x_{2}$ must have different sensitivities towards the two environmental feedback variables (Meszéna et al. 2006). In our framework, this means that the rows of matrix $\mathbf{A}$ in (12) must be different (cf. assumption (A4)). Suppose that strategy $x_{1}$ is strongly sensitive to $E_{1}$ but only weakly to $E_{2}$ (in the sense that the first element of the first row in $\mathbf{A}$ is large and the second element of the first row is small), and the opposite holds for $x_{2}$. Then, by continuity from optimization, $\hat{E}_{1}$ is at a minimum with respect to $x_{1}$; and by the argument above, $\hat{E}_{2}$ is at a maximum with respect to $x_{1}$. Analogously, $\hat{E}_{2}$ is at a minimum with respect to $x_{2}$; and $\hat{E}_{1}$ is at a maximum with respect to $x_{2}$. In other words, $\hat{E}_{1}$ attains a minimum as a function of $x_{1}$ but a maximum as a function of $x_{2}$, i.e., it has a saddle point; and $\hat{E}_{2}$ attains a maximum as a function of $x_{1}$ but a minimum as a function of $x_{2}$, i.e., it too has a saddle point.

In sections 3.3 and 4.1, we explored how the adaptive dynamics of saturated $n$ morphisms connect to the adaptive dynamics of $(n-1)$-morphic populations. The analysis can be extended to the connections also to lower levels of polymorphism, as shown briefly by an example in section 3.3. For $n=2$, the results of section 3.3 neatly separate the roles of the invasion fitness and the embedding model. The invasion fitness alone determines the dimorphic selection gradients and hence the location and stability properties of the dimorphic singularities, as well as the evolutionary stability of a monomorphic singularity given its location. The embedding model influences the location and convergence stability of monomorphic singularities and the admissibility of population dynamical equilibria.

The separation between the invasion fitness and the embedding model motivates the construction method we presented in section 4.2. By changing only the embedding model but not the invasion fitness, we can obtain different pairwise invasibility plots and areas of coexistence to the same trait evolution plot (as in Figure 2). Suppose that the embedding model contains a function that is not part of the invasion fitness. By choosing this function suitably up to first order, we can construct a model such that the invasion 
boundary of the pairwise invasibility plot goes through a given point at a given slope. We used this construction method to obtain different monomorphic singularities without changing the dimorphic selection gradients.

The construction method solves the inverse problem of finding a model to a given outcome, and similar in principle to the so-called critical function analysis, where one constructs trade-off functions such that the model exhibits an evolutionary singularity with required properties (de Mazancourt and Dieckmann 2004; Kisdi 2006; Geritz et al. 2007; Kisdi and Boldin 2013; Kisdi 2015) or some other behaviour such as eco-evolutionary cycles (Kisdi et al. 2013). These construction methods are useful in systematically exploring whether a certain evolutionary outcome, such as evolutionary branching, is possible at all, and if so, under which conditions it occurs. Adamson and Morozov (2014) show a similar inverse problem approach to purely ecological dynamics.

The construction method presented here could be extended to investigate evolutionary phenomena that are not related to evolutionary singularities. A particularly interesting possibility is to study evolution to extinction. Extinction can occur such that the evolution of one strategy drives another strategy extinct, but also via evolutionary suicide, whereby a strategy evolves to its own extinction (Gyllenberg and Parvinen 2001; Gyllenberg et al. 2002; Webb 2003; Boldin and Kisdi, in press). Evolutionary suicide has so far been studied only in monomorphic populations. A catastrophic bifurcation (such as a saddle-node bifurcation) of population dynamics is a necessary condition for evolutionary suicide (Gyllenberg and Parvinen 2001; Gyllenberg et al. 2002), assuming that the invasion fitness remains well defined in the extinct state (see Boldin and Kisdi, in press). In this paper, we used the construction method to obtain a transcritical bifurction of population dynamics (i.e., the invasion boundary), but a similar method could be used to obtain a saddle-node bifurcation of population dynamics. By choosing the slope of the saddle-node bifurcation line such that the evolving dimorphic population crosses it, one can obtain extinction via a catastrophic bifurcation; and by placing the saddle-node bifurcation line on an isocline near a dimorphic singularity (where the selection gradient is parallel to an axis), one can ensure that extinction is due to the evolution of the strategy that goes extinct, i.e., evolutionary suicide occurs.

\section{Acknowledgements}

We thank two anonymous reviewers for comments. This research was financialy supported by the Academy of Finland. 


\section{Appendix A}

In the main text, we assume that the resident population dynamics attain an asymptoticaly stable equilibrium. If the resident system exhibits non-equilibrium dynamics such that the environmental feedbacks vary in time, then the environment is characterized by the functions $t \mapsto E_{1}(t), \ldots, E_{n}(t)$ and is therefore infinite dimensional unless there exists an alternative, finite-dimensional characterization. In this Appendix, we show how such finite-dimensional representations can be found and be used to extend the results of section 3 to some non-equilibrium systems.

In the main text, we assume that the invasion fitness can be written as a function of the invading strategy and numbers $\hat{E}_{1}, \ldots, \hat{E}_{n}$ that represent the environment, such that $\hat{E}_{1}, \ldots, \hat{E}_{n}$ depend on the resident strategies but not on the invading strategy. Assume that the focal strategies have unstructured populations. The invasion fitness of the mutant $x_{m u t}$ is then given by the time average of its population growth rate, i.e., by

$$
\left\langle r\left(x_{m u t}, E_{1}(t), \ldots, E_{n}(t), \psi(t)\right)\right\rangle
$$

where the angle brackets $\langle\cdot\rangle$ denote the time average and we assume that the expectation exists (Metz et al. 1992). We included a new (possibly vector-valued) argument $\psi(t)$ of the population growth rate $r$ to accommodate non-autonomous population dynamics, i.e., the possible effect of temporal fluctuations in the physical environment such as the weather. For the present argument, it does not matter whether the environmental feedbacks vary in time due to endogeneous non-equilibrium dynamics or due to external fluctuations.

If $r\left(x_{m u t}, E_{1}, \ldots, E_{n}, \psi\right)$ is linear in $E_{1}, \ldots, E_{n}$ and in $\psi$, then the invasion fitness simplifies to

$$
\left\langle r\left(x_{m u t}, E_{1}(t), \ldots, E_{n}(t), \psi(t)\right)\right\rangle=r\left(x_{m u t},\left\langle E_{1}(t)\right\rangle, \ldots,\left\langle E_{n}(t)\right\rangle,\langle\psi(t)\rangle\right)
$$

such that the resident population affects the invasion fitness only via the time averages $\left\langle E_{1}(t)\right\rangle, \ldots,\left\langle E_{n}(t)\right\rangle$ of the environmental feedback variables. Equations $(7)$ are then equivalent to

$$
\begin{aligned}
r\left(x_{1},\left\langle E_{1}(t)\right\rangle, \ldots,\left\langle E_{n}(t)\right\rangle,\langle\psi(t)\rangle\right) & =0 \\
& \vdots \\
r\left(x_{n},\left\langle E_{1}(t)\right\rangle, \ldots,\left\langle E_{n}(t)\right\rangle,\langle\psi(t)\rangle\right) & =0
\end{aligned}
$$

and all results of section 3 are valid with $\hat{E}_{i}=\left\langle E_{i}(t)\right\rangle$ for $i=1, \ldots, n$.

There is considerable freedom in how the environmental feedback variables are defined in a given model, and in simple models it is often possible to chose the environmental feedback variables and $\psi$ such that $r$ is linear in them and (26) applies. In the remainder 
of this Appendix, we show several examples based on the host-pathogen-predator model in section 2.

Suppose first that in the model of section 2 , the natural mortality rate $\mu$ depends on time (e.g. varies with the seasons). Since the population growth rate

$$
r\left(\alpha_{m u t}, S, P, \mu\right)=\beta\left(\alpha_{m u t}\right) S-\left(\alpha_{m u t}+\mu+\nu\right)-\left(c+\phi\left(\alpha_{m u t}\right)\right) P
$$

is linear in $S$ and in $P$ and also in $\mu$, we can apply (26) directly with $E_{1}(t)=S(t)$, $E_{2}(t)=P(t)$ and $\psi(t)=\mu(t)$. In other words, we can use $\langle S(t)\rangle$ and $\langle P(t)\rangle$ in place of $\hat{S}$ and $\hat{P}$, respectively, throughout the analysis of the model. In particular, the values of $\langle S(t)\rangle$ and $\langle P(t)\rangle$ in a resident population of strains $\alpha_{1}$ and $\alpha_{2}$ are the same as $\hat{S}\left(\alpha_{1}, \alpha_{2}\right)$ and $\hat{P}\left(\alpha_{1}, \alpha_{2}\right)$ in (5), respectively, in a population at equilibrium with $\mu$ constant at the value of $\langle\mu(t)\rangle$. Therefore, the adaptive dynamics of dimorphic populations would not be affected by the fluctuations (Figure 1 would remain the same). Many simple models of unstructured populations based on mass action are similar to this example. In LotkaVolterra models, it is well known that the time averages of population densities equal to their equilibrium values also if the system does not converge to the equilibrium; the same principle applies here.

Suppose now that predators interfere with each other when hunting for the prey, such that the presence of a second predator decreases the capture rate of the first. A somewhat sloppy way to include predator interference is to replace equation (1) with

$$
\frac{d I_{j}}{d t}=\left[\beta\left(\alpha_{j}\right) S(t)-\left(\alpha_{j}+\mu(t)+\nu\right)-\left(1-e\left(\alpha_{j}\right) P(t)\right)\left(c+\phi\left(\alpha_{j}\right)\right) P(t)\right] I_{j}
$$

where the factor $\left(1-e\left(\alpha_{j}\right) P(t)\right)$ is the probability that a hunting predator is not disturbed, and $e$ may depend on $\alpha_{j}$ because hosts much weakened by the pathogen may be captured faster by the predator, giving less opportunity for interference. This introduces a quadratic term with $P^{2}$ into the population growth rate

$$
r\left(\alpha_{m u t}, S, P, \mu\right)=\beta\left(\alpha_{m u t}\right) S-\left(\alpha_{m u t}+\mu+\nu\right)-\left(c+\phi\left(\alpha_{m u t}\right)\right)+e\left(\alpha_{m u t}\right)\left(c+\phi\left(\alpha_{m u t}\right)\right) P^{2}
$$

In this case, we can define three environmental feedback variables, $E_{1}(t)=S(t), E_{2}(t)=$ $P(t)$ and $E_{3}(t)=[P(t)]^{2} . r$ is linear of each of these, so that (26) applies. Note that through the quadratic term, fluctuations introduce a new feedback, essentially the variance of $P(t)$. It remains to be seen whether three strains can coexist in this system or the number of environmental feedback variables has been inflated without increasing the maximum number of coexisting strains. This depends on the trade-off structure of the pathogen; if $\phi$ and $e$ are constants, then $E_{2}$ and $E_{3}$ combine into a single environmental feedback variable $E(t)=(1-e P(t))(c+\phi) P(t)$, of which $r$ is a linear function, and therefore no more than two strains can coexist.

Suppose next that the natural mortality rate $\mu$ depends also on the total density of the host population, $N$. Then the population growth rate is the same as in (27), but next 
to $E_{1}(t)=S(t)$ and $E_{2}(t)=P(t)$, we have $E_{3}(t)=\mu(N(t), t)$ as a new environmental feedback variable chosen such that (26) applies. In this case, the explicit time-dependence of $\mu$ is absorbed into $E_{3}(t)$.

As a final example, assume that the predator has Holling II functional response and $\mu$ depends only on $t$ again. Then the population growth rate

$r\left(\alpha_{m u t}, S, P, \mu\right)=\beta\left(\alpha_{m u t}\right) S-\left(\alpha_{m u t}+\mu+\nu\right)-\left(c+\phi\left(\alpha_{m u t}\right)\right) \frac{P}{1+T\left(c S+\sum_{k}\left(c+\phi\left(\alpha_{k}\right)\right) I_{k}\right)}$

is linear in the environmental feedback variables $E_{1}(t)=S(t)$ and

$$
E_{2}(t)=\frac{P(t)}{1+T\left(c S(t)+\sum_{k}\left(c+\phi\left(\alpha_{k}\right)\right) I_{k}(t)\right)}
$$

so that (26) applies.

Note that in general, it need not be possible to choose a finite number of environmental feedback variables such that the population growth rate $r$ is linear in them; Geritz et al. (2007) is an example where this is not possible. In these cases, non-equilibrium systems have infinite dimensional feedbacks, which does not constrain the number of coexisting strategies.

\section{Appendix B}

In this Appendix, we first give a sufficient condition for absolute stability of an arbitrary $k$-morphic singularity, where the polymorphism is not necessarily saturated. Next, we show that this condition is always satisfied at a generic evolutionarily stable singularity of a saturated polymorphism.

To describe non-smooth evolutionary trajectories, we consider a differential inclusion approximation of a trait substitution sequence with small mutation steps. Let $\mathbf{x}=\left(x_{1}, \ldots, x_{k}\right)$ be a vector of $k \leq n$ coexisting one-dimensional resident strategies, and let $s_{\mathbf{x}}(y)$ denote the invasion fitness of an initially rare mutant strategy $y$. The selection gradient $\left.\partial_{y} s_{\mathbf{x}}(y)\right|_{y=x_{i}}$ for the resident strategy $x_{i}$ and the evolutionary change $\mathrm{d} x_{i} / \mathrm{d} t$ of the same strategy always have the same sign, i.e.,

$$
\left.\frac{\mathrm{d} x_{i}}{\mathrm{~d} t} \cdot \partial_{y} s_{\mathbf{x}}(y)\right|_{y=x_{i}} \geq 0 \quad \text { for } i=1, \ldots, k
$$

A function $t \mapsto \mathbf{x}(t)=\left(x_{1}(t), \ldots, x_{k}(t)\right)$ is a solution of the differential inclusion (28) if it is continuous, piecewise differentiable and satisfies (28) in every point where it is differentiable. A point $\mathbf{x}^{*}=\left(x_{1}^{*}, \ldots, x_{k}^{*}\right)$ is called absolutely stable if for every open neighbourhood $U$ of $\mathbf{x}^{*}$ there exists an open neighbourhood $V \subset U$ of $\mathbf{x}^{*}$ such that every 
solution of (28) that starts in $V$ will stay in $U$ for all $t \geq 0$. One readily sees that if $\mathbf{x}^{*}$ is absolutely stable, then it is a singular point, i.e.,

$$
\left.\partial_{y} s_{\mathbf{x}}(y)\right|_{y=x_{i}}=0 \quad \text { for } i=1, \ldots, k \text {. }
$$

Linearisation of (28) at a singularity $\mathbf{x}^{*}$ gives

$$
\frac{\mathrm{d} x_{i}}{\mathrm{~d} t} \cdot \sum_{j=1}^{k} b_{i j}\left(x_{j}-x_{j}^{*}\right) \geq 0 \quad \text { for } i=1, \ldots, k .
$$

where

$$
b_{i j}=\partial_{y}^{2} s_{\mathbf{x}}(y) \delta_{i j}+\partial_{x_{j}} \partial_{y} s_{\mathbf{x}}(y)
$$

evaluated for $y=x_{i}^{*}$ and $\mathbf{x}=\mathbf{x}^{*}$, and where $\delta_{i j}$ is the Kronecker delta. Note that $b_{i j}$ in (30) is the same as in (15) of the main text.

Proposition. Let $\mathbf{x}^{*}=\left(x_{1}^{*}, \ldots, x_{k}^{*}\right)$ be a singular point. If the matrix $B=\left(b_{i j}\right)$ is strictly negative diagonally dominant, i.e., if there exists $d_{1}, \ldots, d_{k}>0$ such that

$$
\frac{b_{i i}}{d_{i}}<-\sum_{j \neq i} \frac{\left|b_{i j}\right|}{d_{j}} \quad \forall i
$$

then $\mathbf{x}^{*}$ is absolutely stable for the dynamics given by (29).

Proof. Let $\mathbf{x} \neq \mathbf{x}^{*}$ and take $i_{0} \in\{1, \ldots, k\}$ such that

$$
d_{i_{0}}\left|x_{i_{0}}-x_{i_{0}}^{*}\right|=\max _{j} d_{j}\left|x_{j}-x_{j}^{*}\right|>0
$$

Then in particular $x_{i_{0}}-x_{i_{0}}^{*} \neq 0$, and so we can rewrite (29) as

$$
\frac{\mathrm{d}\left(x_{i_{0}}-x_{i_{0}}^{*}\right)^{2}}{\mathrm{~d} t} \cdot\left(b_{i_{0} i_{0}}+\sum_{j \neq i_{0}} \frac{b_{i_{0} j}\left(x_{j}-x_{j}^{*}\right)}{x_{i_{0}}-x_{i_{0}}^{*}}\right) \geq 0 .
$$

Moreover, from the diagonal dominance (31) and property (32) we have

$$
\begin{gathered}
\frac{b_{i_{0} i_{0}}}{d_{i_{0}}}<-\sum_{j \neq i_{0}} \frac{\left|b_{i_{0} j}\right|}{d_{j}} \leq-\sum_{j \neq i_{0}} \frac{\left|b_{i_{0} j}\right|}{d_{j}} \frac{d_{j}\left|x_{j}-x_{j}^{*}\right|}{d_{i_{0}}\left|x_{i_{0}}-x_{i_{0}}^{*}\right|} \\
\leq-\frac{1}{d_{i_{0}}} \sum_{j \neq i_{0}} \frac{b_{i_{0} j}\left(x_{j}-x_{j}^{*}\right)}{x_{i_{0}}-x_{i_{0}}^{*}}
\end{gathered}
$$

and hence

$$
b_{i_{0} i_{0}}+\sum_{j \neq i_{0}} \frac{b_{i_{0} j}\left(x_{j}-x_{j}^{*}\right)}{x_{i_{0}}-x_{i_{0}}^{*}}<0 .
$$


From this inequality and the differential inclusion (33), we get that $\mathrm{d}\left(x_{i_{0}}-x_{i_{0}}^{*}\right)^{2} / \mathrm{d} t \leq 0$ and hence also $\mathrm{d}\left(d_{i_{0}}\left|x_{i_{0}}-x_{i_{0}}^{*}\right|\right) / \mathrm{d} t \leq 0$, which, by property (32), is equivalent to

$$
\frac{\mathrm{d}}{\mathrm{d} t}\left(\max _{j} d_{j}\left|x_{j}-x_{j}^{*}\right|\right) \leq 0
$$

The nested sets $V_{\varepsilon}=\left\{\left(x_{1}, \ldots, x_{k}\right): \max _{j} d_{j}\left|x_{j}-x_{j}^{*}\right|<\varepsilon\right\}$ for different values of $\varepsilon>0$ are therefore forward invariant for the differential inclusion (29). From this it immediately follows that $\mathbf{x}^{*}$ is absolutely stable. For example, in the definition of absolute stability for given but otherwise arbitrary neighbourhood $U$ any $V=V_{\varepsilon}$ for $\varepsilon>0$ sufficiently small such that $V_{\varepsilon} \subset U$ suffices.

For the special case of $k=2$ coevolving strategies, the conditions for absolute stability have been determined by Matessi and Di Pascuale (1996) and presented by Kisdi (2006) in the form $b_{11}, b_{22}<0$ and $b_{11} b_{22}>\left|b_{12} b_{21}\right|$. These are equivalent to condition (31) for $k=2$. Leimar (2009) considered the absolute stability of monomorphic singularities of multidimensional traits. This is different from the present situation because several traits of the same strategy can be affected by a single mutation whereas the traits of several strategies cannot; the differential inclusion (28) applies to the latter.

Next, we apply the above proposition to saturated polymorphisms $(k=n)$. In the notation of the main text, (30) is

$$
b_{i j}=\partial_{11} r\left(x_{i}, \hat{E}_{1}, \ldots, \hat{E}_{n}\right) \delta_{i j}+\sum_{l=1}^{n} \partial_{1, l+1} r\left(x_{i}, \hat{E}_{1}, \ldots, \hat{E}_{n}\right) \frac{\partial \hat{E}_{l}}{\partial x_{j}}
$$

where $\hat{E}_{1}, \ldots, \hat{E}_{n}$ are evaluated at the singularity and, in accordance with the main text, we have dropped the star that denoted the singularity earlier in this Appendix. By Proposition $2, \frac{\partial \hat{E}_{i}}{\partial x_{j}}=0$ for all $i$ and $j$ at a singularity of a saturated polymorphism, and hence we have $b_{i i}=\partial_{11} r\left(x_{i}, \hat{E}_{1}, \ldots, \hat{E}_{n}\right)$ and $b_{i j}=0$ for all $i \neq j$. At a generic evolutionarily stable singularity, $b_{i i}<0$ (cf. (11)). This implies that (31) is satisfied for any positive $d_{1}, \ldots, d_{n}$, and therefore a generic evolutionarily stable singularity of a saturated polymorphism is also absolutely stable. In the special case of $n=2$, Svennungsen and Kisdi (2009) used the same argument with the stability condition given in Kisdi (2006) to prove that every generic evolutionarily stable dimorphism is absolutely stable. 


\section{Appendix $\mathrm{C}$}

Proof of Proposition 10. We start with proving part (ii) of the proposition. The first step is to derive the limiting value of $\bar{E}_{i}^{\prime}(\epsilon)$ as $\epsilon \rightarrow 0$. From implicit differentiation of (20) with respect to $\epsilon$, we obtain

$$
\mathbf{A}(\epsilon)\left[\begin{array}{c}
\bar{E}_{1}^{\prime}(\epsilon) \\
\vdots \\
\bar{E}_{n}^{\prime}(\epsilon)
\end{array}\right]=\left[\begin{array}{c}
-\xi_{1} \partial_{1} r\left(x_{0}+\epsilon \xi_{1}, \bar{E}_{1}(\epsilon), \ldots, \bar{E}_{n}(\epsilon)\right) \\
-\xi_{2} \partial_{1} r\left(x_{0}+\epsilon \xi_{2}, \bar{E}_{1}(\epsilon), \ldots, \bar{E}_{n}(\epsilon)\right) \\
0 \\
\vdots \\
0
\end{array}\right]
$$

where $\mathbf{A}(\epsilon)$ is as defined in (12), i.e., $a_{i j}=\partial_{j+1} r\left(x_{i}, \bar{E}_{1}(\epsilon), \ldots, \bar{E}_{n}(\epsilon)\right)$, with $x_{1}=x_{0}+\epsilon \xi_{1}$ and $x_{2}=x_{0}+\epsilon \xi_{2}$. Let $\mathbf{A}_{i}(\epsilon)$ denote the matrix obtained by replacing the $i t h$ column of $\mathbf{A}(\epsilon)$ with the vector on the right hand side of (35). By Cramer's rule, the solution of $(35)$ is

$$
\bar{E}_{i}^{\prime}(\epsilon)=\frac{\operatorname{det} \mathbf{A}_{i}(\epsilon)}{\operatorname{det} \mathbf{A}(\epsilon)}
$$

Because the derivatives on the right hand side of (35) are zero at $\epsilon=0$ (cf. (21)), $\mathbf{A}_{i}(0)$ is singular. $\mathbf{A}(0)$ is singular because its first two rows are the same. Hence to take the limit $\epsilon \rightarrow 0$, we use L'Hôpital's rule,

$$
\bar{E}_{i}^{\prime}(0)=\lim _{\epsilon \rightarrow 0} \bar{E}_{i}^{\prime}(\epsilon)=\lim _{\epsilon \rightarrow 0} \frac{\frac{d}{d \epsilon} \operatorname{det} \mathbf{A}_{i}(\epsilon)}{\frac{d}{d \epsilon} \operatorname{det} \mathbf{A}(\epsilon)}
$$

We derive the limit of the numerator and that of the denominator of (36) in turn. We first expand the determinant $\operatorname{det} \mathbf{A}_{i}(\epsilon)$ using its $i$ th column, which is the vector on the right hand side of equation (35):

$$
\begin{array}{r}
\operatorname{det} \mathbf{A}_{i}(\epsilon)=(-1)^{i+1}\left[\xi_{1} \partial_{1} r\left(x_{0}+\epsilon \xi_{1}, \bar{E}_{1}(\epsilon), \ldots, \bar{E}_{n}(\epsilon)\right) \operatorname{det} \mathbf{A}_{1 i}(\epsilon)\right. \\
\left.-\xi_{2} \partial_{1} r\left(x_{0}+\epsilon \xi_{2}, \bar{E}_{1}(\epsilon), \ldots, \bar{E}_{n}(\epsilon)\right) \operatorname{det} \mathbf{A}_{2 i}(\epsilon)\right]
\end{array}
$$

Differentiating with respect to $\epsilon$ and taking the limit $\epsilon \rightarrow 0$, we obtain

$$
\lim _{\epsilon \rightarrow 0} \frac{d}{d \epsilon} \operatorname{det} \mathbf{A}_{i}(\epsilon)=(-1)^{i+1}\left[\left(\xi_{1}^{2}-\xi_{2}^{2}\right) \partial_{11} r+\left(\xi_{1}-\xi_{2}\right) \sum_{l=1}^{n} \partial_{1, l+1} r \bar{E}_{l}^{\prime}(0)\right] \operatorname{det} \mathbf{A}_{1 i}(0)
$$

where all derivatives of $r$ are evaluated at $\left(x_{0}, \bar{E}_{1}(0), \ldots, \bar{E}_{n}(0)\right)$. Here we used that $\partial_{1} r\left(x_{0}, \bar{E}_{1}(0), \ldots, \bar{E}_{n}(0)\right)=0$ (cf. (21)) and because the first two rows of $\mathbf{A}(0)$ are the same, $\mathbf{A}_{1 i}(0)=\mathbf{A}_{2 i}(0)$.

Next, we expand det $\mathbf{A}(\epsilon)$ using its first two rows (i.e., using minors of order 2 rather than elements of a single row):

$$
\operatorname{det} \mathbf{A}(\epsilon)=\sum_{j=1}^{n-1} \sum_{k=j+1}^{n}(-1)^{1+2+j+k} D_{j k}(\epsilon) \operatorname{det} \mathbf{A}_{(1,2),(j, k)}(\epsilon)
$$


where $\mathbf{A}_{(1,2),(j, k)}(\epsilon)$ is obtained from $\mathbf{A}(\epsilon)$ by deleting its first two rows and the $j$ th and $k$ th columns when $n>2$ and $\operatorname{det} \mathbf{A}_{(1,2),(j, k)}(\epsilon)$ is replaced with 1 when $n=2$; and

$$
D_{j k}(\epsilon)=\operatorname{det}\left(\begin{array}{cc}
\partial_{j+1} r\left(x_{0}+\epsilon \xi_{1}, \bar{E}_{1}(\epsilon), \ldots, \bar{E}_{n}(\epsilon)\right) & \partial_{k+1} r\left(x_{0}+\epsilon \xi_{1}, \bar{E}_{1}(\epsilon), \ldots, \bar{E}_{n}(\epsilon)\right) \\
\partial_{j+1} r\left(x_{0}+\epsilon \xi_{2}, \bar{E}_{1}(\epsilon), \ldots, \bar{E}_{n}(\epsilon)\right) & \partial_{k+1} r\left(x_{0}+\epsilon \xi_{2}, \bar{E}_{1}(\epsilon), \ldots, \bar{E}_{n}(\epsilon)\right)
\end{array}\right) .
$$

Note that $D_{j k}(0)=0$ for all $j, k$, and

$$
\begin{aligned}
\lim _{\epsilon \rightarrow 0} \frac{d D_{j k}(\epsilon)}{d \epsilon}= & \left(\xi_{1} \partial_{1, j+1} r+\sum_{l=1}^{n} \partial_{l+1, j+1} r \bar{E}_{l}^{\prime}(0)\right) \partial_{k+1} r \\
& +\left(\xi_{2} \partial_{1, k+1} r+\sum_{l=1}^{n} \partial_{l+1, k+1} r \bar{E}_{l}^{\prime}(0)\right) \partial_{j+1} r \\
& -\left(\xi_{2} \partial_{1, j+1} r+\sum_{l=1}^{n} \partial_{l+1, j+1} r \bar{E}_{l}^{\prime}(0)\right) \partial_{k+1} r \\
& -\left(\xi_{1} \partial_{1, k+1} r+\sum_{l=1}^{n} \partial_{l+1, k+1} r \bar{E}_{l}^{\prime}(0)\right) \partial_{j+1} r \\
= & \left(\xi_{1}-\xi_{2}\right)\left(\partial_{1, j+1} r \partial_{k+1} r-\partial_{1, k+1} r \partial_{j+1} r\right)
\end{aligned}
$$

where all derivatives of $r$ are evaluated at $\left(x_{0}, \bar{E}_{1}(0), \ldots, \bar{E}_{n}(0)\right)$. Using these identities, we obtain

$\lim _{\epsilon \rightarrow 0} \frac{d}{d \epsilon} \operatorname{det} \mathbf{A}(\epsilon)=\left(\xi_{1}-\xi_{2}\right) \sum_{j=1}^{n-1} \sum_{k=j+1}^{n}(-1)^{j+k+1}\left(\partial_{1, j+1} r \partial_{k+1} r-\partial_{1, k+1} r \partial_{j+1} r\right) \operatorname{det} \mathbf{A}_{(1,2),(j, k)}(0)$

Substituting (37) and (38) into (36) and cancelling the factor $\left(\xi_{1}-\xi_{2}\right)$ (recall that $\left.\xi_{1} \neq \xi_{2}\right)$ yields the result

$$
\bar{E}_{i}^{\prime}(0)=\frac{(-1)^{i}\left[\left(\xi_{1}+\xi_{2}\right) \partial_{11} r+\sum_{l=1}^{n} \partial_{1, l+1} r \bar{E}_{l}^{\prime}(0)\right] \operatorname{det} \mathbf{A}_{1 i}}{\sum_{j=1}^{n-1} \sum_{k=j+1}^{n}(-1)^{j+k}\left[\partial_{1, j+1} r \partial_{k+1} r-\partial_{1, k+1} r \partial_{j+1} r\right] \operatorname{det} \mathbf{A}_{(1,2),(j, k)}}
$$

for $i=1, \ldots, n$, where all derivatives of $r$ are evaluated at $\left(x_{0}, \bar{E}_{1}(0), \ldots, \bar{E}_{n}(0)\right)$. We assume that the non-degeneracy condition

$$
\sum_{j=1}^{n-1} \sum_{k=j+1}^{n}(-1)^{j+k}\left[\partial_{1, j+1} r \partial_{k+1} r-\partial_{1, k+1} r \partial_{j+1} r\right] \operatorname{det} \mathbf{A}_{(1,2),(j, k)} \neq 0
$$

holds.

(39) is a system of linear equations for $\bar{E}_{i}^{\prime}(0)$. With the particular choice $\xi_{2}=-\xi_{1}$, (39) is homogeneous and therefore $\bar{E}_{i}^{\prime}(0)=0$ for all $i$. This corresponds to the fact that $\hat{E}_{i}\left(x_{1}, \ldots, x_{n}\right)$ is invariant under the permutation of its arguments (the labelling of the strategies is arbitrary), so that the the environmental feedback contours of Figure 1 are symmetric about the diagonal. 
If $\bar{E}_{i}^{\prime}(0)=0$ holds for all $\xi_{1}, \xi_{2}$ for some $i$, then $\partial_{11} r\left(x_{0}, \bar{E}_{1}(0), \ldots, \bar{E}_{n}(0)\right)$ must be zero for (39) to hold. This means that $x_{0} \in X^{c}$. Furthermore, (39) is then homogeneous, and therefore $\bar{E}_{i}^{\prime}(0)=0$ holds for all $i$, so that each environmental feedback attains a critical point as a function of $x_{1}$ and $x_{2}$. Conversely, if $x_{0} \in X^{c}$, then (39) is homogeneous and it follows immediately that $\bar{E}_{i}^{\prime}(0)=0$ for all $i$ and for all $\xi_{1}, \xi_{2}$, so that all environmental feedbacks attain a critical point on the diagonal as functions of $x_{1}$ and $x_{2}$. This proves part (ii) of Proposition 10.

To prove part (i), take a first order Taylor expansion of (9) to obtain

$$
\begin{aligned}
& \partial_{1} r\left(x_{0}+\epsilon \xi_{1}, \bar{E}_{1}(\epsilon), \ldots, \bar{E}_{n}(\epsilon)\right)=\left[\xi_{1} \partial_{11} r+\sum_{l=1}^{n} \partial_{1, l+1} r \bar{E}_{l}^{\prime}(0)\right] \epsilon+O\left(\epsilon^{2}\right) \\
& \partial_{1} r\left(x_{0}+\epsilon \xi_{2}, \bar{E}_{1}(\epsilon), \ldots, \bar{E}_{n}(\epsilon)\right)=\left[\xi_{2} \partial_{11} r+\sum_{l=1}^{n} \partial_{1, l+1} r \bar{E}_{l}^{\prime}(0)\right] \epsilon+O\left(\epsilon^{2}\right)
\end{aligned}
$$

where all derivatives of $r$ on the right hand side are evaluated at $\left(x_{0}, \bar{E}_{1}(0), \ldots, \bar{E}_{n}(0)\right)$. Choose $x_{0}=\frac{x_{1}+x_{2}}{2}$ such that $\xi_{2}=-\xi_{1}$. With this choice, it follows from equation (39) that $\bar{E}_{l}^{\prime}(0)=0$ for all $l$. Then, up to order $\epsilon$, the vector of selection gradients of the first two strategies is

$$
\left[\begin{array}{c}
1 \\
-1
\end{array}\right] \xi_{1} \partial_{11} r\left(x_{0}, \bar{E}_{1}(0), \ldots, \bar{E}_{n}(0)\right) \epsilon
$$

These two selection gradients vanish simultaneously, and vanish if and only if $x_{0} \in X^{c}$. This proves part (i) of Proposition 10.

Proof of Proposition 12. When $n=2$, the vector in (40) is the vector of all selection gradients. This vector is perpendicular to the diagonal $x_{2}=x_{1}$, and its direction is de-

termined by the sign of $\partial_{11} r\left(x_{0}, \bar{E}_{1}(0), \ldots, \bar{E}_{n}(0)\right)$.

\section{Appendix D}

In this Appendix, we prove Propositions 13 and 14 concerning the common points of isoclines and invasion boundaries.

Proof of Proposition 13 for the general case $n \geq 2$. Let the $n$-vector $\mathbf{x}_{m j}$ denote the vector obtained from $\mathbf{x}=\left(x_{1}, \ldots, x_{n}\right)$ by replacing its $m$ th entry with the value of $x_{j}$. The point $\mathbf{x}_{m j}$ is therefore on the diagonal $x_{m}=x_{j}$. With a slight abuse of notation, let $\bar{E}_{i}\left(\mathbf{x}_{m j}\right)$ denote the limiting value of $\hat{E}_{i}(\mathbf{x})$ on the diagonal. If $\mathbf{x}$ is on the $x_{j}$-isocline of the $n$-morphism, then, by Proposition $9, \hat{E}_{i}(\mathbf{x})=\bar{E}_{i}\left(\mathbf{x}_{m j}\right)$ for $i=1, \ldots, n$. If $\mathbf{x}$ is also on the $x_{m}$-invasion boundary, then, by Lemma 2 in section 4.1 , the diagonal point $\mathbf{x}_{m j}$ must also be on the $x_{m}$-invasion boundary. This implies that the diagonal point is a point of the $x_{j}$-isocline of the $(n-1)$-morphic population. Conversely, if the diagonal point $\mathbf{x}_{m j}$ is an $x_{j}$-isocline point of the $(n-1)$-morphism, then it is on the $x_{m}$-invasion boundary of the $n$-morphism. By Lemma 2, every point $\mathbf{x}$ which differs from $\mathbf{x}_{m j}$ only 
in its $x_{m}$-coordinate and at which $\hat{E}_{i}(\mathbf{x})=\bar{E}\left(\mathbf{x}_{m j}\right)$ holds for $i=1, \ldots, n$ must also be on the $x_{m}$-invasion boundary. By Proposition 9 , these points are on the $x_{j}$-isocline of the $n$-morphism.

Proof of Proposition 14. Define the column vector $\mathbf{x}_{\neg \mathbf{m}}=\left(x_{1}, \ldots, x_{m-1}, x_{m+1}, \ldots, x_{n}\right)^{T}$ as the $(n-1)$-vector obtained from $\left(x_{1}, \ldots, x_{n}\right)$ by deleting $x_{m}$, define $\mathbf{N}_{\neg \mathbf{m}}$ analogously, and let $\mathbf{Z}=\left(Z_{1}, \ldots, Z_{k}\right)^{T}$. We can then rewrite equations (23) in the form

$$
\mathbf{G}\left(\mathbf{x}_{\neg \mathbf{m}}, x_{m}, \mathbf{N}_{\neg \mathbf{m}}, \mathbf{Z}\right)=\mathbf{0}
$$

where $\mathbf{G}\left(\mathbf{x}_{\neg \mathbf{m}}, x_{m}, \mathbf{N}_{\neg \mathbf{m}}, \mathbf{Z}\right) \in \mathbb{R}^{n+k}$ contains

$$
\begin{aligned}
& G_{i}\left(\mathbf{x}_{\neg \mathbf{m}}, x_{m}, \mathbf{N}_{\neg \mathbf{m}}, \mathbf{Z}\right)= \\
& \quad=F_{i}\left(x_{1}, \ldots, x_{n}, \hat{E}_{1}(\mathbf{x}), \ldots, \hat{E}_{n}(\mathbf{x}), N_{1}, \ldots, N_{m-1}, 0, N_{m+1}, \ldots, N_{n}, Z_{1}, \ldots, Z_{k}\right)
\end{aligned}
$$

Note that $F_{i}$ does not depend on its $m$ th argument due to assumption (A3), but still depends on $x_{m}$ via $\hat{E}_{1}(\mathbf{x}), \ldots, \hat{E}_{n}(\mathbf{x})$, which are given by the solution of $(7)$ irrespectively of whether $N_{m}$ is zero or not.

First we determine the hyperplane tangent to the $x_{m}$-invasion boundary at an arbitrary point. Implicit differentiation of (41) yields

$$
[\mathbf{B}, \mathbf{C}]\left[\begin{array}{c}
d \mathbf{x}_{\neg \mathbf{m}} \\
d x_{m} \\
d \mathbf{N}_{\neg \mathbf{m}} \\
d \mathbf{Z}
\end{array}\right]=\mathbf{B}\left[d \mathbf{x}_{\neg \mathbf{m}}\right]+\mathbf{C}\left[\begin{array}{c}
d x_{m} \\
d \mathbf{N}_{\neg \mathbf{m}} \\
d \mathbf{Z}
\end{array}\right]=\mathbf{0}
$$

where the blocks $\mathbf{B}, \mathbf{C}$ of the Jacobian matrix are

$$
\mathbf{B}=\left[\frac{\partial \mathbf{G}}{\partial \mathbf{x}_{\neg \mathbf{m}}}\right] \quad \text { and } \quad \mathbf{C}=\left[\frac{\partial \mathbf{G}}{\partial x_{m}}, \frac{\partial \mathbf{G}}{\partial \mathbf{N}_{\neg \mathbf{m}}}, \frac{\partial \mathbf{G}}{\partial \mathbf{Z}}\right]
$$

By Cramer's rule, (42) yields

$$
d x_{m}=\frac{\operatorname{det}\left[-\mathbf{B} d \mathbf{x}_{\neg \mathbf{m}}, \frac{\partial \mathbf{G}}{\partial \mathbf{N}_{\neg \mathbf{m}}}, \frac{\partial \mathbf{G}}{\partial \mathbf{Z}}\right]}{\operatorname{det}[\mathbf{C}]}
$$

The hyperplane tangent to the $x_{m}$-invasion boundary at a given point is the hyperplane $a_{1} x_{1}+\ldots+a_{n} x_{n}=c$ with the coefficients chosen such that $\sum_{i} a_{i} d x_{i}=0$ for any choice of $d \mathbf{x}_{\neg \mathbf{m}}$ and with $d x_{m}$ from (43). By choosing the unit vectors $d \mathbf{x}_{\neg \mathbf{m}}=\mathbf{e}_{\mathbf{i}}$ and $d \mathbf{x}_{\neg \mathbf{m}}=\mathbf{e}_{\mathbf{j}}$ with $i, j \neq m$, we obtain

$$
\frac{a_{i}}{a_{j}}=\frac{\operatorname{det}\left[\mathbf{B e}_{i}, \frac{\partial \mathbf{G}}{\partial \mathbf{N}_{\neg \mathbf{m}}}, \frac{\partial \mathbf{G}}{\partial \mathbf{Z}}\right]}{\operatorname{det}\left[\mathbf{B e}_{j}, \frac{\partial \mathbf{G}}{\partial \mathbf{N}_{\neg \mathbf{m}}}, \frac{\partial \mathbf{G}}{\partial \mathbf{Z}}\right]}
$$


which determines all coefficients except $a_{m}$ up to a constant. Let the $(n-1)$ column vector $\mathbf{a}_{\neg \mathbf{m}}$ contain these coefficients. $a_{m}$ is then given by

$$
a_{m}=-\left[\mathbf{a}_{\neg \mathbf{m}}^{T} d \mathbf{x}_{\neg \mathbf{m}}\right] \frac{\operatorname{det}[\mathbf{C}]}{\operatorname{det}\left[-\mathbf{B} d \mathbf{x}_{\neg \mathbf{m}}, \frac{\partial \mathbf{G}}{\partial \mathbf{N}_{\neg \mathbf{m}}}, \frac{\partial \mathbf{G}}{\partial \mathbf{Z}}\right]}
$$

The hyperplane tangent to the $x_{m}$-invasion boundary at a given point is determined by (44) and (45), with all derivatives evaluated at the point of tangency.

Suppose now that the point $\left(x_{1}, \ldots, x_{n}\right)$ is both on the $x_{m}$-invasion boundary and on the $x_{m}$-isocline. By Proposition 1 , we then have $\frac{\partial \hat{E}_{1}}{\partial x_{m}}=\ldots=\frac{\partial \hat{E}_{n}}{\partial x_{m}}=0$, and this implies $\frac{\partial \mathbf{G}}{\partial x_{m}}=\mathbf{0}$. Because the first column of $\mathbf{C}$ is zero, its determinant vanishes and we get $a_{m}=0$ in (45). Hence the normal of the $x_{m}$-invasion boundary, $\left(a_{1}, \ldots, a_{n}\right)$, is perpendicular to the $m$ th unit vector.

\section{Appendix E}

Here we derive $\hat{h}_{1}\left(x_{1}, x_{2} ; \kappa\right)$ in equation (25) of the main text. Although this derivation is in the same spirit as the one in Appendix D, we detail it here since it is easier to follow it when presented explicitly.

Let $\hat{N}_{1}\left(x_{1}, x_{2}\right)$ and $\hat{Z}_{1}\left(x_{1}, x_{2}\right), \ldots, \hat{Z}_{k}\left(x_{1}, x_{2}\right)$ denote the values of respectively $N_{1}$ and $Z_{1}, \ldots, Z_{k}$ that satisfy the $k+1$ equations $F_{2}=\ldots=F_{k+2}=0$ in (24) and recall the definition $\hat{f}\left(x_{1}, x_{2}\right)=f\left(x_{1}, x_{2}, \hat{E}_{1}\left(x_{1}, x_{2}\right), \hat{E}_{2}\left(x_{1}, x_{2}\right), \hat{N}_{1}\left(x_{1}, x_{2}\right), 0, \hat{Z}_{1}\left(x_{1}, x_{2}\right), \ldots, \hat{Z}_{k}\left(x_{1}, x_{2}\right)\right)$. By implicit differentiation of (24) with respect to $x_{1}$ and using that, by assumption (A3), $\partial_{2} \tilde{F}_{1}=\partial_{2} F_{2}=\ldots=\partial_{2} F_{k+2}=0$ on the invasion boundary, we obtain

$$
\begin{gathered}
\partial_{1} \tilde{F}_{1}+\sum_{i=1}^{2} \partial_{i+2} \tilde{F}_{1}\left(\frac{\partial \hat{E}_{i}}{\partial x_{1}}+\frac{\partial \hat{E}_{i}}{\partial x_{2}} \kappa\right)+\partial_{5} \tilde{F}_{1} y_{0}+\sum_{i=1}^{k} \partial_{6+i} \tilde{F}_{1} y_{i}+ \\
+\partial_{k+7} \tilde{F}_{1} h^{\prime}\left(\hat{f}\left(x_{1}, x_{2}\right)\right)\left(\frac{\partial \hat{f}}{\partial x_{1}}+\frac{\partial \hat{f}}{\partial x_{2}} \kappa\right)=0 \\
\partial_{1} F_{2}+\sum_{i=1}^{2} \partial_{i+2} F_{2}\left(\frac{\partial \hat{E}_{i}}{\partial x_{1}}+\frac{\partial \hat{E}_{i}}{\partial x_{2}} \kappa\right)+\partial_{5} F_{2} y_{0}+\sum_{i=1}^{k} \partial_{6+i} F_{2} y_{i}=0 \\
\vdots \\
\partial_{1} F_{k+2}+\sum_{i=1}^{2} \partial_{i+2} F_{k+2}\left(\frac{\partial \hat{E}_{i}}{\partial x_{1}}+\frac{\partial \hat{E}_{i}}{\partial x_{2}} \kappa\right)+\partial_{5} F_{k+2} y_{0}+\sum_{i=1}^{k} \partial_{6+i} F_{k+2} y_{i}=0
\end{gathered}
$$

where $\kappa=\frac{d x_{2}}{d x_{1}}$ is the slope of the invasion boundary, $y_{0}=\frac{\partial \hat{N}_{1}}{\partial x_{1}}+\frac{\partial \hat{N}_{1}}{\partial x_{2}} \kappa, y_{i}=\frac{\partial \hat{Z}_{i}}{\partial x_{1}}+$ $\frac{\partial \hat{Z}_{i}}{\partial x_{2}} \kappa$ for $i=1, \ldots, k$, and all derivatives of $\tilde{F}_{1}, F_{2}, \ldots, F_{k+2}$ are evaluated at the arguments 
$\left(x_{1}, x_{2}, \hat{E}_{1}\left(x_{1}, x_{2}\right), \hat{E}_{2}\left(x_{1}, x_{2}\right), \hat{N}_{1}\left(x_{1}, x_{2}\right), 0, \hat{Z}_{1}\left(x_{1}, x_{2}\right), \ldots, \hat{Z}_{k}\left(x_{1}, x_{2}\right)\right)$ and, in the case of $\tilde{F}_{1}$, $\hat{h}_{0}\left(x_{1}, x_{2}\right)$. These equations are satisfied when $h^{\prime}\left(\hat{f}\left(x_{1}, x_{2}\right)\right)=\hat{h}_{1}\left(x_{1}, x_{2} ; \kappa\right)$ where

$$
\hat{h}_{1}\left(x_{1}, x_{2} ; \kappa\right)=-\frac{\partial_{1} \tilde{F}_{1}+\sum_{i=1}^{2} \partial_{i+2} \tilde{F}_{1}\left(\frac{\partial \hat{E}_{i}}{\partial x_{1}}+\frac{\partial \hat{E}_{i}}{\partial x_{2}} \kappa\right)+\partial_{5} \tilde{F}_{1} y_{0}+\sum_{i=1}^{k} \partial_{6+i} \tilde{F}_{1} y_{i}}{\partial_{k+7} \tilde{F}_{1}\left(\frac{\partial \hat{f}}{\partial x_{1}}+\frac{\partial \hat{f}}{\partial x_{2}} \kappa\right)}
$$

with the values of $y_{0}, \ldots, y_{k}$ that satisfy the last $k+1$ equations of (46). (Since the denominator of (47) vanishes for $\kappa=-\frac{\partial \hat{f}}{\partial x_{1}} / \frac{\partial \hat{f}}{\partial x_{2}}$, this particular slope of the invasion boundary cannot be achieved.)

In the remainder of this Appendix, we consider the special cases when (i) the point $\left(x_{1}, x_{2}\right)$ is on the $x_{2}$-isocline and (ii) $\left(x_{1}, x_{2}\right)=\left(x_{0}, x_{0}\right)$ with $x_{0} \in X^{c}$.

If the $x_{2}$-invasion boundary intersects the $x_{2}$-isocline away from the diagonal, then, by Proposition 14, the invasion boundary must be tangent to a vertical line; hence one cannot choose the function $h$ such that the invasion boundary would have an arbitrary slope $\kappa$. Consider (46) as a system of $k+2$ linear equations for the unknowns $\kappa, y_{0}, y_{1}, \ldots, y_{n}$. The point $\left(x_{1}, x_{2}\right)$ is on the $x_{2}$-isocline if and only if $\frac{\partial \hat{E}_{1}}{\partial x_{2}}=\frac{\partial \hat{E}_{2}}{\partial x_{2}}=0$ (see Proposition 1). As we show in the next paragraph, this implies $\frac{\partial \hat{f}}{\partial x_{2}}=0$. As a result, the coefficient of $\kappa$ vanishes in each equation of (46), i.e., the matrix of this linear system is singular, and therefore the implicit function theorem does not apply.

To show that $\frac{\partial \hat{E}_{1}}{\partial x_{2}}=\frac{\partial \hat{E}_{2}}{\partial x_{2}}=0$ indeed implies $\frac{\partial \hat{f}}{\partial x_{2}}=0$, note that the terms containing $\kappa$ in the last $k+1$ equations of (46) all vanish, so that these equations can be solved for $y_{0}, \ldots, y_{n}$ independently of $\kappa$. Since $y_{0}=\frac{\partial \hat{N}_{1}}{\partial x_{1}}+\frac{\partial \hat{N}_{1}}{\partial x_{2}} \kappa$ and $y_{i}=\frac{\partial \hat{Z}_{i}}{\partial x_{1}}+\frac{\partial \hat{Z}_{i}}{\partial x_{2}} \kappa$, this implies $\frac{\partial \hat{N}_{1}}{\partial x_{2}}=0$ and $\frac{\partial \hat{Z}_{i}}{\partial x_{2}}=0$ for $i=1, \ldots, k$. By definition, the derivative $\frac{\partial \hat{f}}{\partial x_{2}}$ is given by

$$
\frac{\partial \hat{f}}{\partial x_{2}}=\partial_{2} f+\partial_{3} f \frac{\partial \hat{E}_{1}}{\partial x_{2}}+\partial_{4} f \frac{\partial \hat{E}_{2}}{\partial x_{2}}+\partial_{5} f \frac{\partial \hat{N}_{1}}{\partial x_{2}}+\sum_{i=1}^{k} \partial_{6+i} f \frac{\partial \hat{Z}_{i}}{\partial x_{2}}
$$

Hence all but the first terms vanish when $\frac{\partial \hat{E}_{1}}{\partial x_{2}}=\frac{\partial \hat{E}_{2}}{\partial x_{2}}=0$; and since the derivative is evaluated at $\left(x_{1}, x_{2}, \hat{E}_{1}\left(x_{1}, x_{2}\right), \hat{E}_{2}\left(x_{1}, x_{2}\right), \hat{N}_{1}\left(x_{1}, x_{2}\right), 0, \hat{Z}_{1}\left(x_{1}, x_{2}\right), \ldots, \hat{Z}_{k}\left(x_{1}, x_{2}\right)\right), \partial_{2} f$ is zero by assumption (A7).

Suppose now that we wish to have a monomorphic singularity at a point $x_{0} \in X^{c}$, i.e., we take $\left(x_{1}, x_{2}\right)=\left(x_{0}, x_{0}\right)$. By the definition of the set $X^{c}, C_{00}=0$ at such a singularity, i.e., it is at a bifurcation point between being uninvadable and invadable. By Proposition 10 , the limiting values of the equilibrium feedbacks attain a critical point at $\left(x_{0}, x_{0}\right)$. Hence the above argument applies with $\hat{E}_{i}\left(x_{1}, x_{2}\right)$ substituted with its limiting value at all points, and we obtain, as above, that the matrix of the linear system that determines $\kappa$ for any given function $h$ is singular. Therefore generically there is no solution for $\kappa$, 
and the invasion boundary is tangent to a vertical line at the singularity.

\section{References}

Adamson M. W. and A. Yu. Morozov. 2014. Bifurcation analysis of models with uncertain function specification: How should we proceed? Bull. Math. Biol. 76: 12181240.

Boldin B. and Kisdi E. 2012. On the evolutionary dynamics of pathogens with direct and environmental transmission. Evolution 66: 2514-2527.

Boldin B. and Kisdi E. Evolutionary suicide through a non-catastrophic bifurcation. J. Math. Biol., in press.

de Mazancourt C. and Dieckmann U. 2004. Trade-off geometries and frequency-dependent selection. Am. Nat. 164: 765-778.

Dercole F., Ferriere R. and Rinaldi S. 2010. Chaotic red queen coevolution in three-species food chains. Proc. R. Soc. B 277: 2321-2330.

Dercole F., Irisson J.-O. and Rinaldi S. 2003. Bifurcation analysis of a prey-predator coevolution model. SIAM J. Appl. Math. 63: 1378-1391.

Dieckmann U. and Law R. 1996. The dynamical theory of coevolution: A derivation from stochastic ecological processes. J. Math. Biol. 34: 579-612.

Diekmann O., Gyllenberg M., Huang H., Kirkilionis M., Metz J. A. J. and Thieme H. R. 2001. On the formulation and analysis of general deterministic structured population models. II. Nonlinear theory. J. Math. Biol. 43: 157-189.

Diekmann O., Gyllenberg M. and Metz J. A. J. 2003. Steady state analysis of structured population models. Theor. Pop. Biol. 63: 309-338.

Diekmann O., Gyllenberg M., Metz J. A. J. and Thieme H. R. 1998. On the formulation and analysis of general deterministic structured population models. I. Linear theory. Journal of Mathematical Biology 36: 349-388.

Durinx M., Metz J. A. J. and Meszéna G. 2008. Adaptive dynamics for physiologically structured population models. J. Math. Biol. 56: 673-742.

Eshel I. 1983. Evolutionary and continuous stability. J. theor. Biol. 103: 99-111. 
Gavrilets S. 1997. Coevolutionary chase in exploiter-victim systems with polygenic characters. J. theor. Biol. 186: 527-534.

Geritz S. A. H. 2005. Resident-invader dynamics and the coexistence of similar strategies. J. Math. Biol. 50: 67-82.

Geritz S. A. H., Gyllenberg M., Jacobs F. and Parvinen K. 2002. Invasion dynamics and attractor inheritance. J. Math. Biol. 44: 548-560.

Geritz S. A. H. and Kisdi E. 2000. Adaptive dynamics in diploid, sexual populations and the evolution of reproductive isolation. Proc. R. Soc. Lond. B 267: 1671-1678.

Geritz S. A. H. Kisdi E., Meszéna G. and Metz J.A.J. 1998. Evolutionarily singular strategies and the adaptive growth and branching of the evolutionary tree. Evol. Ecol. 12: $35-57$.

Geritz S. A. H. Kisdi E. and Yan P. 2007. Evolutionary branching and long-term coexistence of cycling predators: Critical function analysis. Theor. Pop. Biol. 71: 424-435.

Geritz S. A. H., Metz J. A. J., Kisdi E. and Meszéna G. 1997. Dynamics of adaptation and evolutionary branching. Phys. Rev. Letters 78: 2024-2027.

Geritz S. A. H., van der Meijden E. and Metz J. A. J. 1999. Evolutionary dynamics of seed size and seedling competitive ability. Theor. Pop. Biol. 55: 324-343.

Gyllenberg M. and Meszéna. 2005. On the impossibility of coexistence of infinitely many strategies. J. Math. Biol. 50: 133-160.

Gyllenberg M., Metz J. A. J. and Service R. 2011. When do optimisation arguments make evolutionary sense? In: Chalub, F.A.C. and Rodrigues, J.R. (eds.): The Mathematics of Darwins Legacy, Birkhuser, Springer Basel, pp. 233-268.

Gyllenberg M. and Parvinen K. 2001. Necessary and sufficient conditions for evolutionary suicide. Bull. Math. Biol. 63: 981-993.

Gyllenberg M., Parvinen K. and Dieckmann U. 2002. Evolutionary suicide and evolution of dispersal in structured metapopulations. J. Math. Biol. 45: 79-105.

Gyllenberg M. and Service R. 2011. Necessary and sufficient conditions for the existence of an optimisation principle in evolution. J. Math. Biol. 62: 359-369.

Khibnik A. I. and Kondrashov A. S. 1997. Three mechanisms of Red Queen dynamics. Proc. R. Soc. Lond. B 264: 1049-1056. 
Kisdi E. 1999. Evolutionary branching under asymmetric competition. J. theor. Biol. 197: $149-162$.

Kisdi E. 2006. Trade-off geometries and the adaptive dynamics of two co-evolving species. Evol. Ecol. Res. 8: 959-973.

Kisdi E. 2015. Construction of multiple trade-offs to obtain arbitrary singularities of adaptive dynamics. J. Math. Biol. 70: 1093-1117.

Kisdi E. and Boldin B. 2013. A construction method to study the role of incidence in the adaptive dynamics of pathogens with direct and environmental transmission. J. Math. Biol. 66: 1021-1044.

Kisdi E., Geritz S. A. H. and Boldin B. 2013. Evolution of pathogen virulence under selective predation: A construction method to find eco-evolutionary cycles. J. theor. Biol. 339: $140-150$.

Leimar O. 2009. Multidimensional convergence stability. Evol. Ecol. Res. 11: 191-208.

Levin S. 1970. Community equilibria and stability, and an extension of the competitive exclusion principle. Am. Nat. 104: 413-423.

MacArthur R. and Levins R. 1964. Competition, habitat selection, and character displacement in a patchy environment. Proc. Natl. Acad. Sci. USA 51: 1207-1210.

Marrow P., Dieckmann U. and Law R. 1996. Evolutionary dynamics of predator-prey systems: An ecological perspective. J. Math. Biol. 34: 556-578.

Matessi C. and Di Pasquale C. 1996. Long-term evolution of multilocus traits. J. Math. Biol. 34: 613-653.

Mathias A. and Kisdi E. 2002. Adaptive diversification of germination strategies. Proc. R. Soc. Lond. B 269: 151-156.

Maynard Smith J. 1982. Evolution and the theory of games. Cambridge University Press, Cambridge.

Meszéna G., Gyllenberg M., Pásztor L. and Metz J. A. J. 2006. Competitive exclusion and limiting similarity: A unified theory. Theor. Pop. Biol. 69: 68-87.

Meszéna G., Kisdi E., Dieckmann U., Geritz S. A. H. and Metz J. A. J. 2001. Evolutionary optimisation models and matrix games in the unified perspective of adaptive 
dynamics. Selection 2: 193-210.

Metz J. A. J. and Geritz S. A. H. Frequency dependence 3.0: An attempt at codifying the evolutionary ecology perspective. J. Math. Biol., in press.

Metz J. A. J., Geritz S. A. H., Meszéna G., Jacobs F. J. A. and van Heerwaarden J. S. 1996a. Adaptive dynamics, a geometrical study of the consequences of nearly faithful reproduction. In: S. J. van Strien \& S. M. Verduyn Lunel (eds): Stochastic and spatial structures of dynamical systems. North Holland, Amsterdam, pp. 183-231.

Metz J. A. J., Mylius S. and Diekmann O. 1996b. When does evolution optimize? On the relation between types of density dependence and evolutionarily stable life history parameters. IIASA Working Paper WP-96-004, available online: http://webarchive.iiasa.ac.at/Admin/PUB/Documents/WP-96-004.pdf

Metz J. A. J., Mylius S. and Diekmann O. 2008. When does evolution optimize? Evol. Ecol. Res. 10: 629-654.

Metz J. A. J., Nisbet R. M. and Geritz S. A. H. 1992. How should we define 'fitness' for general ecological scenarios? Trends Ecol. Evol. 7:198-202.

Morozov A. \& A. Best. 2012. Predation on infected host promotes evolutionary branching of virulence and pathogens' biodiversity. J. theor. Biol. 307: 29-36.

Mylius S. D. and Diekmann O. 1995. On evolutionarily stable life histories, optimization and the need to be specific about density dependence. Oikos 74: 218-224.

Svennungsen T. O. and Holen O. H. 2007. The evolutionary stability of automimicry. Proc. R. Soc. B 274: 2055-2062.

Svennungsen T. and Kisdi E. 2009. Evolutionary branching of virulence in a singleinfection model. J. theor. Biol. 257: 408-418.

Webb C. 2003. A complete classification of Darwinian extinction in ecological interactions. Am. Nat. 161: 181-205.

Weigang H. C. and Kisdi E. 2015. Evolution of dispersal under a fecundity-dispersal trade-off. J. theor. Biol. 371: 145-153. 


\section{Correction}

This is the Author Version of the correction published in the Journal of Mathematical Biology. The final publication of the correction is available at Springer Verlag via https://doi.org/10.1007/s00285019-01398-5.

In the original publication, Proposition 4 is mistaken. The proposition considers models with two environmental feedback variables, $E_{1}$ and $E_{2}$, and two resident strategies with trait values $x_{1}$ and $x_{2}$. The original proposition claimed that if the invasion fitness $r\left(x, E_{1}, E_{2}\right)$ is uniformly monotonic in $E_{i}(i=1$ or 2$)$, then the equilibrium value of this same feedback as a function of the trait values, $\hat{E}_{i}\left(x_{1}, x_{2}\right)$, has a saddle point at a generic evolutionarily stable singularity. In fact, it is not $\hat{E}_{i}\left(x_{1}, x_{2}\right)$ but the other feedback that has a saddle point under the conditions stated in Proposition 4 of the original publication. The corrected proposition and its proof appear below. The error does not affect the rest of the article.

In most models that we encounter, the invasion fitness is uniformly monotonic in all feedback variables (this also pertains to the example presented in section 2 of the original publication). In this case, both feedbacks have a saddle point and the correction of the proposition has no practical consequence; yet the proof needs to be corrected.

Proposition 4. Suppose there are two environmental feedback variables and two coexisting resident strategies $(n=2)$. If the invasion fitness is uniformly monotonic in the environmental feedback variable $E_{1}$, then $\hat{E}_{2}\left(x_{1}, x_{2}\right)$ has a saddle point at a generic evolutionarily stable dimorphic singularity; and vice versa, if the invasion fitness is uniformly monotonic in $E_{2}$, then $\hat{E}_{1}\left(x_{1}, x_{2}\right)$ has a saddle point at a generic evolutionarily stable dimorphic singularity. Specifically, the environmental feedback at the saddle is maximized as a function of one trait value and minimized as a function of the other.

Proof. For $n=2$, equation (16) of the original article reads

$$
\mathbf{A}\left[\begin{array}{c}
\frac{\partial^{2} \hat{E}_{1}}{\partial x_{j} \partial x_{k}} \\
\frac{\partial^{2} \hat{E}_{2}}{\partial x_{j} \partial x_{k}}
\end{array}\right]=\left\{\begin{array}{cl}
-\partial_{11} r\left(x_{j}, \hat{E}_{1}, \hat{E}_{2}\right) \mathbf{e}_{j} & \text { if } k=j \\
\mathbf{0} & \text { otherwise }
\end{array}\right.
$$

where $j, k \in\{1,2\}$ and the matrix $\mathbf{A}$ has elements $A_{i j}=\partial_{j+1} r\left(x_{i}, \hat{E}_{1}, \hat{E}_{2}\right)$. In particular, with $k=j$, we have

$$
\mathbf{A}\left[\begin{array}{c}
\frac{\partial^{2} \hat{E}_{1}}{\partial x_{1}^{2}} \\
\frac{\partial^{2} \hat{E}_{2}}{\partial x_{1}^{2}}
\end{array}\right]=\left[\begin{array}{c}
-\partial_{11} r\left(x_{1}, \hat{E}_{1}, \hat{E}_{2}\right) \\
0
\end{array}\right]
$$

and

$$
\mathbf{A}\left[\begin{array}{c}
\frac{\partial^{2} \hat{E}_{1}}{\partial x_{2}^{2}} \\
\frac{\partial^{2} E_{2}}{\partial x_{2}^{2}}
\end{array}\right]=\left[\begin{array}{c}
0 \\
-\partial_{11} r\left(x_{2}, \hat{E}_{1}, \hat{E}_{2}\right)
\end{array}\right]
$$


for $j=1$ and for $j=2$, respectively. Using Cramer's rule, we thus obtain

$$
\begin{aligned}
\frac{\partial^{2} \hat{E}_{1}}{\partial x_{1}^{2}} & =\frac{-\partial_{11} r\left(x_{1}, \hat{E}_{1}, \hat{E}_{2}\right) \cdot \partial_{3} r\left(x_{2}, \hat{E}_{1}, \hat{E}_{2}\right)}{\operatorname{det} \mathbf{A}} \\
\frac{\partial^{2} \hat{E}_{1}}{\partial x_{2}^{2}} & =\frac{\partial_{11} r\left(x_{2}, \hat{E}_{1}, \hat{E}_{2}\right) \cdot \partial_{3} r\left(x_{1}, \hat{E}_{1}, \hat{E}_{2}\right)}{\operatorname{det} \mathbf{A}}
\end{aligned}
$$

and

$$
\begin{aligned}
\frac{\partial^{2} \hat{E}_{2}}{\partial x_{1}^{2}} & =\frac{\partial_{11} r\left(x_{1}, \hat{E}_{1}, \hat{E}_{2}\right) \cdot \partial_{2} r\left(x_{2}, \hat{E}_{1}, \hat{E}_{2}\right)}{\operatorname{det} \mathbf{A}} \\
\frac{\partial^{2} \hat{E}_{2}}{\partial x_{2}^{2}} & =\frac{-\partial_{11} r\left(x_{2}, \hat{E}_{1}, \hat{E}_{2}\right) \cdot \partial_{2} r\left(x_{1}, \hat{E}_{1}, \hat{E}_{2}\right)}{\operatorname{det} \mathbf{A}}
\end{aligned}
$$

The second derivatives $\partial_{11} r\left(x_{1}, \hat{E}_{1}, \hat{E}_{2}\right)$ and $\partial_{11} r\left(x_{2}, \hat{E}_{1}, \hat{E}_{2}\right)$ are both negative at a generic evolutionarily stable singularity. If the invasion fitness is uniformly monotonic in $E_{2}$, then the factors $\partial_{3} r\left(x_{2}, \hat{E}_{1}, \hat{E}_{2}\right)$ and $\partial_{3} r\left(x_{1}, \hat{E}_{1}, \hat{E}_{2}\right)$ in equations (1a) and (1b) have the same sign. Due to the minus sign that appears in (1a) but not in (1b), $\frac{\partial^{2} \hat{E}_{1}}{\partial x_{1}^{2}}$ and $\frac{\partial^{2} \hat{E}_{1}}{\partial x_{2}^{2}}$ have opposite signs. Together with Proposition 2 of the orignal article, this shows that $\hat{E}_{1}\left(x_{1}, x_{2}\right)$ is maximized as a function of one trait value and minimized as a function of the other, i.e., it has a saddle point at a generic evolutionarily stable dimorphic singularity. Similarly, if the invasion fitness is uniformly monotonic in $E_{1}$, then the factors $\partial_{2} r\left(x_{2}, \hat{E}_{1}, \hat{E}_{2}\right)$ and $\partial_{2} r\left(x_{1}, \hat{E}_{1}, \hat{E}_{2}\right)$ in equations (2a) and (2b) have the same sign, so that $\frac{\partial^{2} \hat{E}_{2}}{\partial x_{1}^{2}}$ and $\frac{\partial^{2} \hat{E}_{2}}{\partial x_{2}^{2}}$ have opposite signs; $\hat{E}_{2}\left(x_{1}, x_{2}\right)$ is therefore maximized as a function of one trait value and minimized as a function of the other. 